\title{
Le techno-complexe hoabinhien en Asie du Sud-est continentale : $L$ 'histoire d'un galet qui cache la forêt
}

\author{
Hubert Forestier ${ }^{1}$, Heng Sophady ${ }^{2}$, Vincenzo Celiberti ${ }^{3}$ \\ 1. Muséum national d'Histoire naturelle, UMR 7194 CNRS-MNHN-UPVD, Institut de Paléontologie Humaine, \\ 1, rue René-Panhard, 75013 Paris, France. Email: hubforestier@gmail.com \\ 2. Ministry of Culture and Fine Arts, 227 Kbal Thnal, Preah Norodom Boulevard, Sangkat Tonle Bassac, Khan \\ Chamkar Mon Phnom Penh 12305, Cambodia. Email: hsophady@yahoo.com \\ 3. Université de Perpignan Via Domitia, UMR 7194 CNRS-MNHN-UPVD, Centre Européen de Recherches \\ Préhistoriques, Avenue Léon-Jean Grégory, 66720 Tautavel, France. \\ Email: vincenzo.celiberti@cerptautavel.com
}

\section{Résumé :}

La préhistoire du Sud-est asiatique se caractérise par un matériel lithique dont les chaînes opératoires restent encore peu connues ou mal décrites. Cette méconnaissance s'explique par l'éloignement géographique de ces régions tropicales vis à vis des problématiques préhistoriques occidentales développées depuis maintenant deux siècles. La préhistoire de l'Extrême-Orient est complexe, originale, surprenante parfois paradoxale car en marge des grandes lignées techniques connues ailleurs pour la période concernée, celle qui a vu l'avènement de l'Homme anatomiquement moderne. Cette préhistoire régionale d'environ 2 millions de $\mathrm{km}^{2}$ que l'on nomme l'Asie du Sud-est péninsulaire ou continentale renvoie à l'élaboration d'une science en mouvement dont la construction est toujours d'actualité. En se heurtant à la thèse classique de l'évolution comme à celle du progrès technique, le Hoabinhien bouscule les règles et les repères en préhistoire. Il se situe aux antipodes du modèle classique (Eurasiatique, Africain) de développement des outils de pierre selon l'enrichissement et l'allègement progressif de l'outillage depuis la pebble culture jusqu'au Néolithique. C'est précisément sur cette singularité que porte notre réflexion à propos d'un phénomène technique unique qui reste difficile à positionner sur l'axe évolutif des industries tel qu'il existe ailleurs dans le monde ou en Asie de l'Est (Chine, Corée, Japon). La régularité et l'homogénéité des formes d'outils façonnées sur galet dans un vaste espace et sur une durée record de près de 30000 ans sont les caractéristiques principales de ce technocomplexe peu orthodoxe qui interroge la capacité cognitive d'Homo sapiens en écosystème tropical humide. Mais aussi, la question de la nature des liens existants entre l'homme et ses productions lithiques ou la place occupée par la technique de la pierre taillée lors du développement humain dans cette région hors d’Europe.

A cette monotone pérennité d'outils sur galet se greffe l'absence d'outils lithiques pointus (pointe, extrémité apicale, outil à bords saillants convergents, etc.) tels qu'ils se rencontrent partout ailleurs chez les groupes de chasseurs-cueilleurs qu'ils soient du Paléolithique supérieur ou (sub-)actuels.

C’est pourquoi la stabilité de ces outillages sur galet cacherait tout un pan de complexité inconnu du domaine technique comme l'insaisissable travail des matières dures d'origine animale mais surtout végétale non conservées à ce jour dans les niveaux archéologiques. Le cheminement du minéral vers la

Published by the School of History, Classics and Archaeology, University of Edinburgh ISSN: 2055-0472. URL: http://journals.ed.ac.uk/lithicstudies/

This work is licensed under a Creative Commons Attribution 2.5 UK: Scotland License. 
question du végétal renvoie à la nécessité de compléter la «boîte à outils » des artisans préhistoriques hoabinhiens par des objets pointus. Connue à ce jour qu’à travers les données de l'ethnographie, la " civilisation du végétal » conduit tout naturellement à réfléchir sur l’importance de ce matériau périssable dans le bagage outillé des derniers chasseurs-cueilleurs de la forêt tropicale d'Asie du Sudest continentale. Autrement dit, la possibilité d'une autre existence technique en équilibre avec le milieu extérieur.

Après avoir mis en avant l'originalité du phénomène culturel hoabinhien au regard de l'impact de la recherche en préhistoire et en paléoanthropologie dans les régions du Sud-est asiatique, cet article présentera d'un point de vue strictement qualitatif, les chaînes opératoires du techno-complexe hoabinhien. Faciès industriel sur galet qui caractérise la principale culture technique des chasseurscueilleurs du Paléolithique final d'Asie du Sud-est entre environ 30000 et 5000 ans BP. Plus largement des précisions seront apportées d'une part sur l'inachèvement informatif du phénomène lithique en tant que donnée archéologique et d'autre part, sur son dépassement en tant que phénomène. Il s'agira donc de repenser l'envers de la dialectique lithique-lignic c'est-à-dire les objets du végétal à jamais disparus, à la lumière des objets de pierre taillée seuls conservés comme marqueurs de temps, de technique, d'espace et d'absence.

Mots-clés : Asie du Sud-Est; Paléolithique supérieur final; Hoabinhien; chaîne opératoire; galet taillé; chasseurs-cueilleurs; homme moderne

\section{Une géographie particulière}

Le terme Southeast Asia fut prononcé pour la première fois dans les années 1940 par les Britanniques lors de la guerre contre l'expansion japonaise en Indochine et en Insulinde. Cette entité géographique complexe recouvre près de 4 millions de $\mathrm{km}^{2}$. Prise en étau entre deux autres Asies, l'une méridionale et l'autre orientale délimitant respectivement le monde indien et le monde chinois, l'Asie du Sud-est a une physiographie où se mêlent des environnements tropicaux et océaniques (Figure 1). Par sa situation géographique l'Asie du Sud-est constitue une synthèse au sens propre comme au sens figuré d'une extension physique de ce que l'on nomme l'Orient, entité symétriquement opposée à l'Occident. Une région de carrefour, de creuset et de transition, qualifiée « d'angle de l'Asie » par P. Mus en 1977 qui voit l'étirement de la vaste masse continentale eurasiatique du sud de la Chine, de la Birmanie, de la Thaïlande, du Laos, du Vietnam et du Cambodge vers le monde océanique via la Malaisie, puis l'Indonésie et les Philippines. A l'exception peut-être pour les très vieux outils datés de 800000 ans attribués à Homo erectus mis au jour en Indonésie dans le dôme de Sangiran à Java Central (Sémah 2001; Sémah et al. 1992; 2014), la préhistoire paléolithique de l’Asie du Sud-est et l'évolution de ses assemblages lithiques restent largement méconnues par grand nombre de préhistoriens européens. Il en est de même de sa géographie et de ses frontières.

Aux confins de l'Asie de l'Est et du Sud, le Sud-est asiatique se scinde en deux parties d'environ 2 millions de $\mathrm{km}^{2}$ chacune, constituées d'un vaste socle continental et d'une guirlande insulaire volcanique s'émiettant en équilibre instable entre les profondes fosses des océans Indien et Pacifique jusqu'à la plateforme d'Arafura rassemblant le bloc Papouasie Nouvelle-Guinée - Australie - Tasmanie (Grande Australie ou Sahul). L'ensemble géographique dit "continental » qui nous intéresse au premier chef, rassemble un étirement péninsulaire structuré autour d'un double emboitement à la fois indochinois et malais, limité au Sud par le Golfe de Bengale, le détroit de Malacca et la mer de Chine méridionale. Cette zone géographique pourrait se résumer en trois mots : chaleurs, pluies et forêts. Cette Asie est une Asie tropicale, forestière, chaude et humide sans hiver à mousson, structurée par de grands fleuves aux riches deltas (Irrawadi, Salaween, Chao Praya, Mekong, Sông Hông, etc.). 


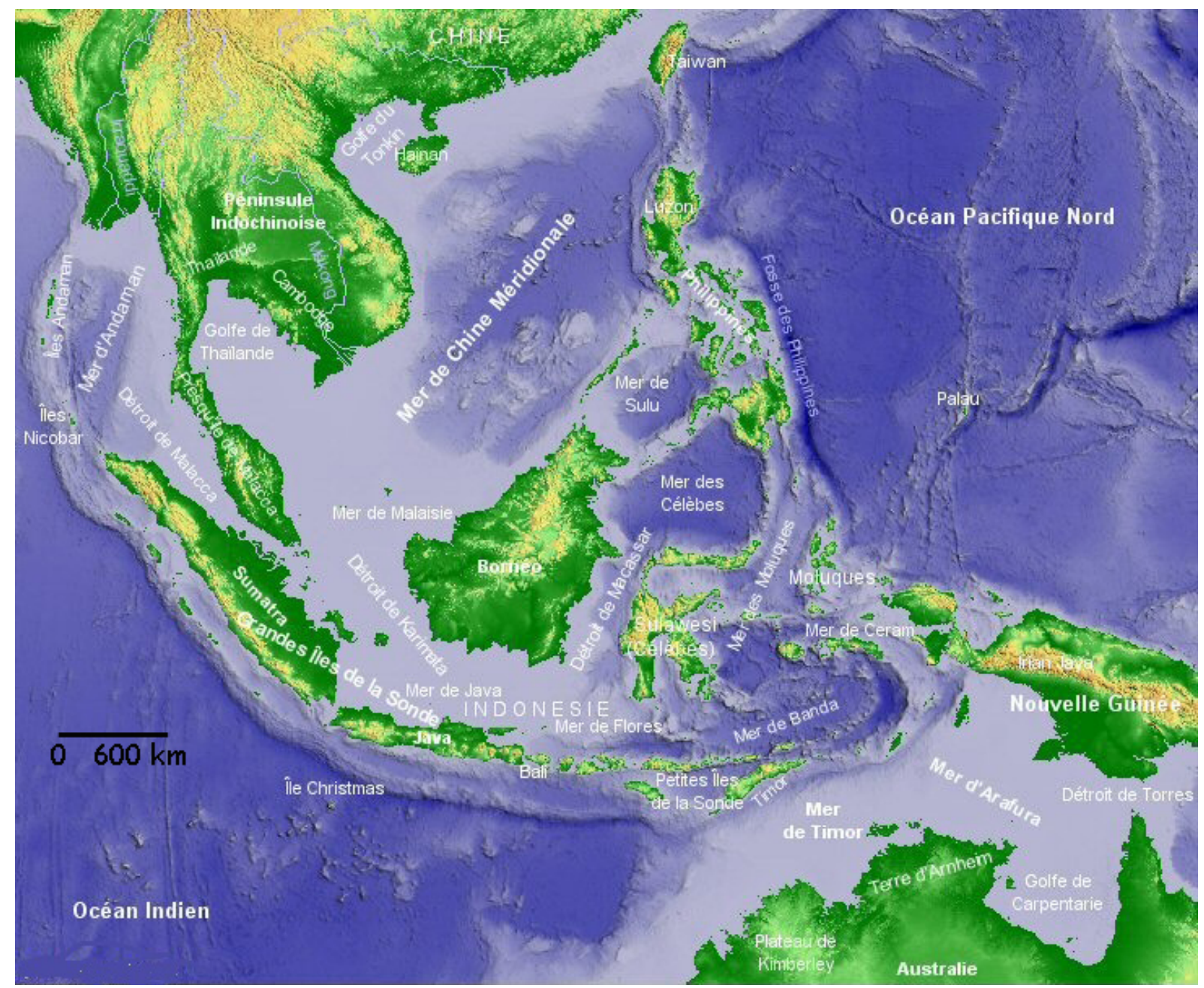

Figure 1. Localisation géographique de l’Asie du Sud-est.

Figure 1. Geographical location of Southeast Asia.

\section{Un enjeu paléoanthropologique}

La biogéographie riche en contrastes issus de l'opposition archipel ou continent confère à l'Asie du Sud-est une place privilégiée et originale dans l'enregistrement des peuplements du passé. A la fois terre d'accueils, d'exils et d'écueils au cours du Quaternaire, elle a joué à certaines époques le rôle de cul-de-sac pour plusieurs espèces animales dont l'homme. Certaines s'y sont éteintes (Homo erectus) ; alors que pour d'autres, ce fût un creuset et un centre de radiations bio-culturelles vers d'autres régions.

L'Asie du Sud-est s'affiche comme un pôle inégalé de diversification humaine y compris pour notre sous-espèce moderne (Homo sapiens sapiens) sur l'ensemble de l'Ancien Monde. La question y est débattue de l'existence de plusieurs souches locales d'Homo sapiens ou bien, d'une évolution régionale d'origine est-africaine suivie par une expansion globale et massive (par vagues successives) avec un remplacement systématique des anciennes populations endémiques. Si le débat est loin d'être clos, le bilan actuel des recherches paléoanthropologiques fait apparaître deux, voire trois et même plus récemment quatre espèces humaines en Asie du Sud-est: Homo erectus, Homo sapiens, Homo soloensis (Zeitoun et al. 2010) et Homo floresiensis (Brown et al. 2004). Deux théories (modèle multirégional versus modèle unicentriste) s'affrontent à propos des modalités de peuplement de l'Ancien Monde qui voient leurs origines et leurs dénouements en Asie (Zeitoun 2004). Le premier modèle favoriserait une évolution biologique sur place d'Homo erectus en Homo sapiens dans toutes les régions du monde et, notamment en Asie et en particulier en Asie du 
Sud-est ; quant au second modèle, il penche plutôt pour le remplacement des Homo erectus locaux par des Homo sapiens à la faveur de vagues migratrices de populations originaires d'Afrique orientale.

Préludes à la compréhension d'un monde asiatique actuel connu pour être le plus peuplé et un des plus diversifiés de notre planète, ces problématiques paléoanthropologiques cruciales nécessiteraient d'être menées en tenant compte des changements climatiques globaux et de leurs expressions locales. En effet, à grande échelle temporelle, il apparaît que ce sont les changements environnementaux qui commandent les phénomènes biologiques évolutifs majeurs avant que l'homme amorce une émancipation partielle et se singularise ensuite par des différences culturelles et techniques.

A l'instar de l'anthropologie biologique qui recense au moins quatre espèces d'hommes fossiles en Asie du Sud-est (Zeitoun 2009), l'archéologie préhistorique devrait répertorier plusieurs types d'industries lithiques selon le modèle habituel d'évolution par allègement de l'outillage et des ruptures technologiques (galet, biface, éclat, lame, lamelle, microlithe, etc.). Couramment observé dans d'autres aires géographiques (Europe de l'Ouest et Europe Centrale, Afrique, Proche-Orient, etc.), ce modèle à visée universelle établi par les préhistoriens occidentaux ne convient pas pour l'Asie du Sud-est. En effet, le paradigme occidental (Pigeot 1991) qui présente un découpage cognitif argumenté de l'évolution technique en plusieurs stades suivant l'évolution phylogénique des taxons humains sur la longue période du Paléolithique (inférieur, moyen et supérieur), ne s’y applique pas.

En conséquence, sur un plan strictement culturel, l'Asie du Sud-est se distingue du modèle évolutionniste des outils de pierre taillée depuis la pebble culture jusqu'au Néolithique, traduit et radicalisé en modes 0 à 5 (Clark 1969) ou plus récemment, en modes A à I (Shea 2013), puisque la totalité de ses assemblages resterait à un stade initial (le galet). C'est précisément sur cette singularité que porte notre réflexion autour du techno-complexe hoabinhien, principale culture technique de l'homme moderne dans cette immense région du monde.

\section{La préhistoire du Sud-est asiatique : une anomalie singulière ?}

Depuis une quinzaine d'années, le matériel lithique des sites préhistoriques de cette aire géographique nous a permis de nous interroger sur la nature et le sens de l'évolution technique des outils de pierre taillée émergeant dans un contexte d'environnement humide, tropical et forestier. Le phénomène hoabinhien ne respecte en rien le modèle globalisant proposé (voir supra). Ce qui nous a conduit à réfléchir sur l'universalisme du paradigme communément accepté et par conséquent sur sa validité compte tenu de l'étendue de notre région d'étude. Nous devrons également nous interroger sur l'existence ou non de facteurs de variabilité, de complexité et de complémentarité des systèmes de production de débitage et de façonnage locaux. Les chaînes opératoires hoabinhiennes comprises entre le Pléistocène supérieur final et le milieu de l'Holocène (entre 30000 et 5000 ans BP) identifiées dans ces régions tropicales et récemment réétudiées sur un plan technologique, constitueront la matière à partir de laquelle s’appuiera cet article.

Si l'Europe du Paléolithique supérieur affiche au même moment et pendant la même durée des successions rapides et individualisables de traditions culturelles sur près de 30000 ans (i.e. le Paléolithique supérieur stricto sensu), c'est loin d'être le cas pour l'Asie du Sud-est où l'histoire des techniques serait aux antipodes géographiques et paradigmatiques. L' "évolution buissonnante » des industries lithiques d'Europe occidentale dont parlait F. Bordes (1950), ne concernerait ici que les fossiles humains (!) quand une stagnation technique se caractériserait par la pérennité d’outillages réalisés sur galets. Par conséquent, au moment où l'homme moderne et ses industries, pour beaucoup d'entre elles lamino-lamellaires, sont 
présents quasiment partout dans le monde entre 24 000-18 000 BP (LGM), le scénario sud-est asiatique est tout autre comme d'ailleurs d'autres aires géographiques en zone tropicale et intertropicale (Forestier 2014). En effet, on y observe une tradition technique composée généralement d'outils sommaires, frustres sur galets invariables et homogènes comme c'est le cas pour le Hoabinhien.

\section{Le poids de l'histoire}

Si la préhistoire est encore une discipline discrète et peu pratiquée au Cambodge, au Vietnam ou au Laos à cause des funestes évènements (guerres et génocides) qui ont entaché l'histoire contemporaine de l'Indochine, elle y a pourtant été exportée très tôt en accompagnant les missions scientifiques de l'Europe coloniale. Dès la fin du XIX ${ }^{\mathrm{ème}}$ siècle, au moment même où E. Dubois découvrait le Pithécanthrope à Java en 1891 et inventait la Paléoanthropologie, la préhistoire du Sud-est asiatique comme celle du Cambodge retenait déjà l'intérêt de grands préhistoriens français tel E. Cartailhac (1877).

En Indochine, le Service Géologique fondé en 1899 par H. Mansuy et E. Patte favorisa les recherches en stratigraphie et paléontologie qui ont été suivies dans la première moitié du $X X^{\text {ème }}$ siècle par J. Fromaget au Laos et plus récemment dans les années 1960 par E. Saurin et J. P. Carbonell sur l'ensemble du Quaternaire indochinois puis au Cambodge par R. et C. Mourer (Mourer 1994).

Pendant l'entre-deux guerres et juste après, les chercheurs britanniques M.W. Tweedie et G. de Sieveking ont fait avancer les recherches en Malaisie notamment sur le faciès hoabinhien du site de Gua Cha (Sieveking 1954). D’autres comme H. de Terra ont mené des recherches pionnières au Myanmar, pays au potentiel considérable qui n’a plus connu de travaux significatifs en préhistoire paléolithique depuis cette époque (Movius 1948).

Après la seconde guerre mondiale, plusieurs recherches d'ampleur ont été menées comme par exemple dans la grotte de Niah à Kalimantan (Bornéo) par T. Harrisson (1957; 1963; 1970) alors qu'en Indonésie et en Thaïlande débutaient les premiers grands travaux de synthèse sur la chronologie des industries lithiques de H. R. Van Heekeren (1972), R. P. Soejono (1962) et P. Sørensen (1976).

Parmi toutes ces personnalités masculines, nous devons la découverte et l'invention du terme « Hoabinhien » à une femme d'exception, Madeleine Colani (1927) qui a légué dans les années 1940 les premières collections lithiques provenant du Nord Vietnam au Musée de l’Homme à Paris (Figure 2).

\section{Naissance d'une singularité régionale}

Né au Nord Vietnam dans les années 1930 d’une femme et taillé par un Homo sapiens, le terme Hoabinhien est ainsi apparu pour la première fois à la suite des travaux pionniers de Madeleine Colani. Alors chercheuse en géologie et en préhistoire au Service Géologique de l'Indochine puis rattachée à l'École française d'Extrême-Orient, elle a prospecté et fouillé près d'une soixantaine de cavités dans les provinces calcaires nord vietnamiennes (Tonkin) : sites de Da Phuc, Lang Gam, Lang Vanh, Lang Tieng dans la province de Hoa Binh située à environ $70 \mathrm{~km}$ à l'Ouest de Hanoi (Figure 3) mais également Ha Nam, Thanh Hoa, Quang Binh, etc. (Colani 1927; 1929a; 1929b). La province de Hoa-Binh située au Sud-Ouest du Fleuve Rouge, devenue la région éponyme du Hoabinhien, concentre le plus grand nombre de grottes contenant des assemblages d'outils préhistoriques façonnés sur galets plats. Ces assemblages comptent un grand nombre d'outils unifaciaux dans les séries archéologiques, le reste étant constitué d’éclats parfois retouchés (Figure 2). 

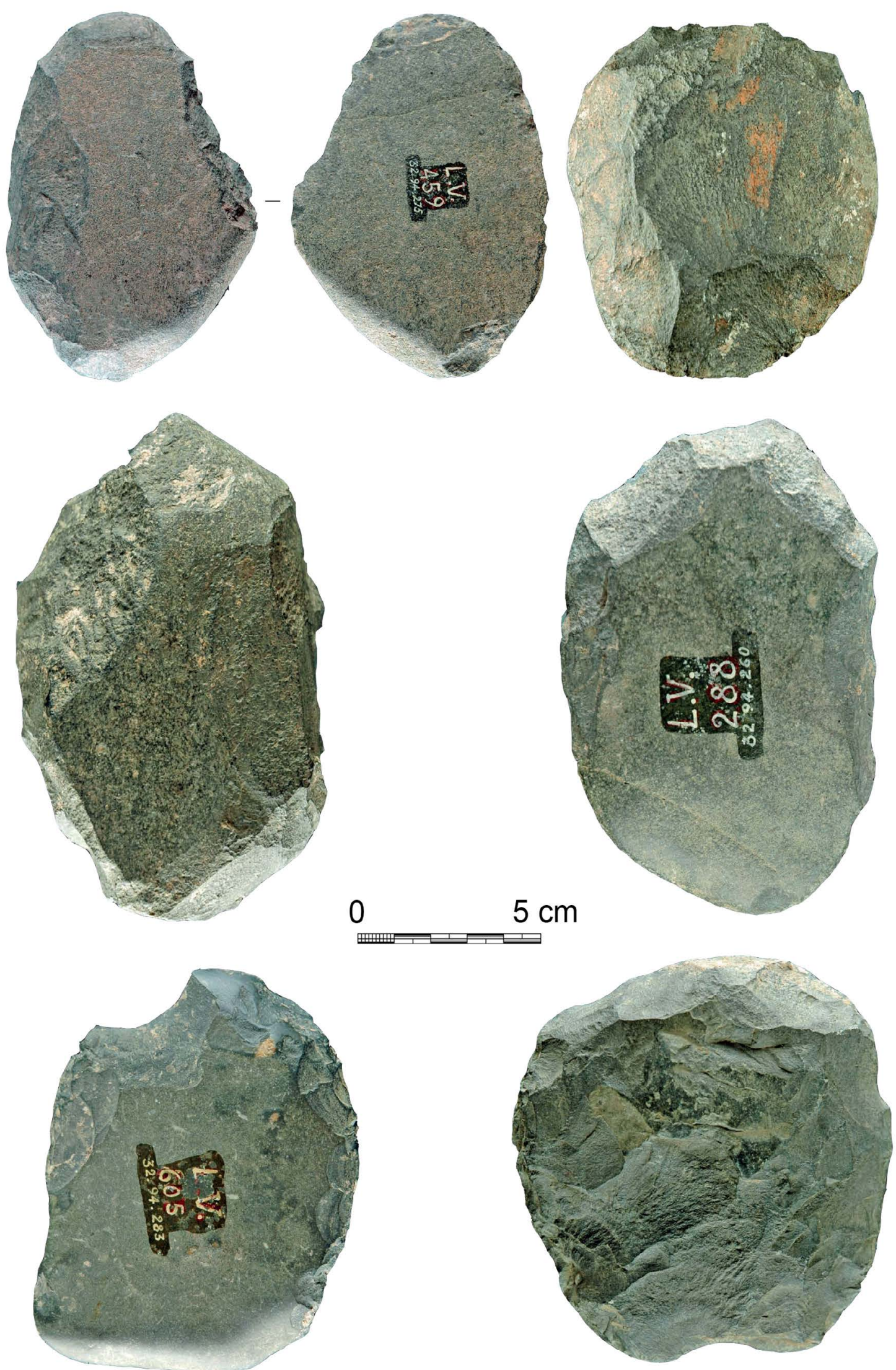

Figure 2. Outils typiques hoabinhiens, Nord Vietnam (Coll. Colani 1932, Musée de l'Homme, Paris) (H. Forestier).

Figure 2. Typical Hoabinhian pebble tools from North Vietnam (Colani Coll. 1932, Musée de l’Homme, Paris) (H. Forestier). 

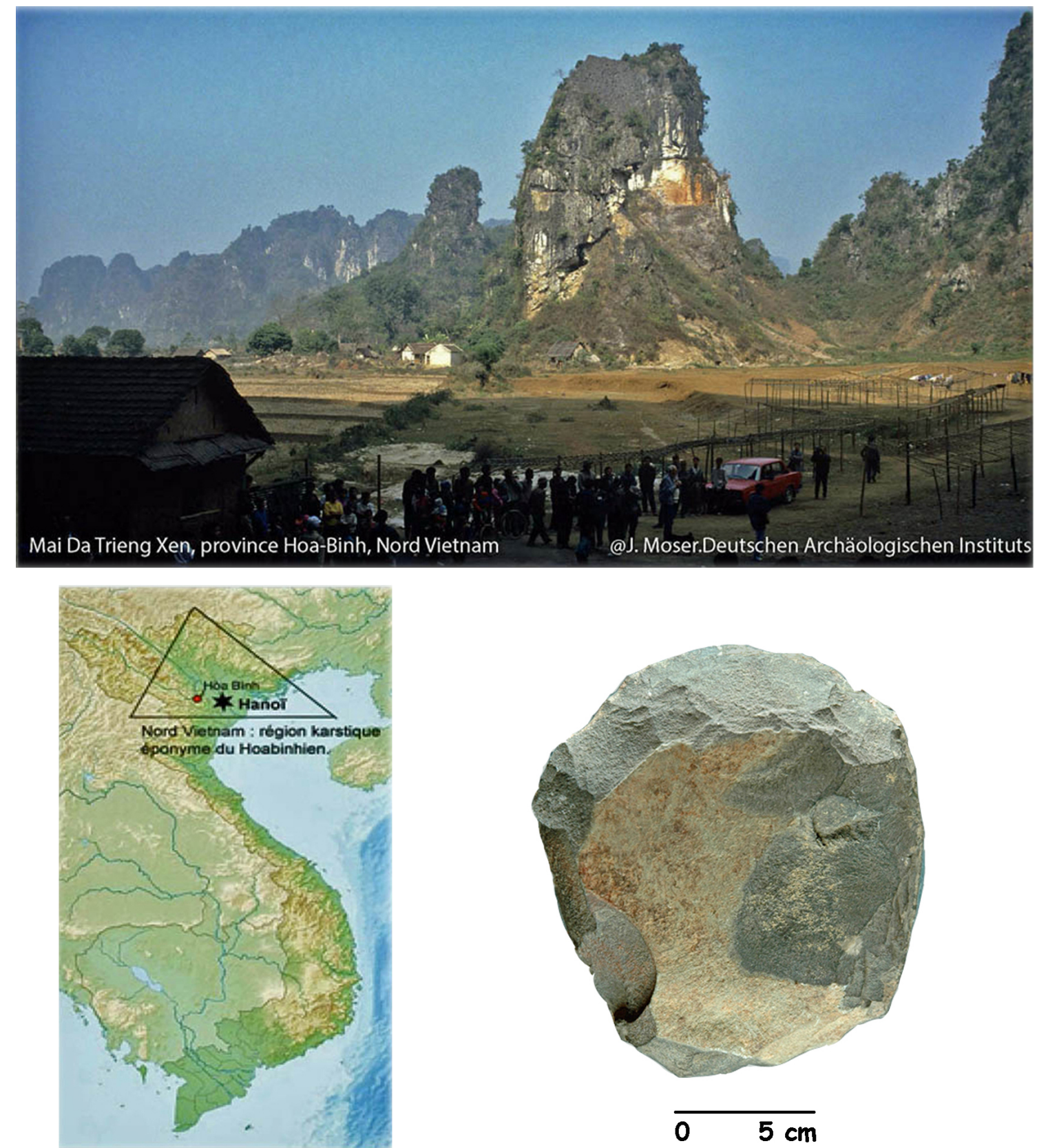

Figure 3. Localisation de l'aire éponyme du Hoabinhien avec le site Mai Da Trieng Xen (Province Hoa-Binh, Nord Vietnam) (photo d'après J. Moser).

Figure 3. Localisation of the eponymous Hoabinhian area with Mai Da Trieng Xen site (Hoa-Binh Province, North Vietnam) (photo after J. Moser).

Dès 1932 le premier congrès de préhistoire d’Extrême-Orient consacra ces découvertes pionnières en ratifiant le terme Hoabinhien. Une première définition typo-morphologique fut avancée décrivant ce faciès comme des "instruments généralement taillés avec des types assez variés et de façonnement assez primitif. Il se caractérise par des outils souvent taillés sur une face, des percuteurs, des pièces à grande section subtriangulaire, des disques, des haches courtes et des instruments amigdaloïdes » (Collectif 1932: 11).

Le terme Hoabinhien sert depuis à nommer les industries lithiques préhistoriques des chasseurs-cueilleurs de la fin du Paléolithique d'Asie du Sud-est continentale et plus discrètement celles de la partie péninsulaire malaise (Majid 2003; Rahman 2002) ou de la région nord de l’île de Sumatra en Indonésie (Forestier et al. 2005a) (Figure 4). 


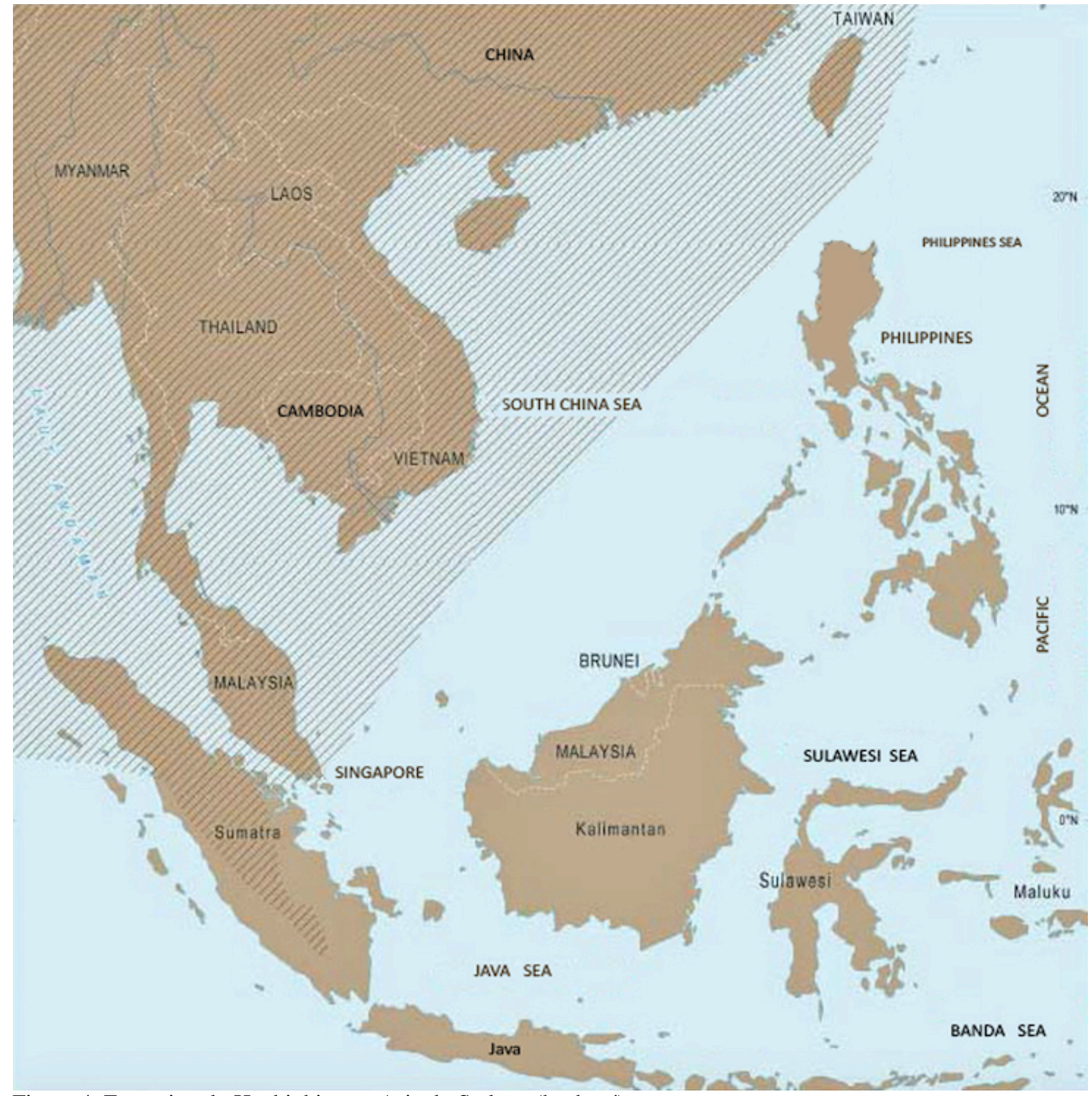

Figure 4. Extension du Hoabinhien en Asie du Sud-est (hachuré).

Figure 4. Extension of Hoabinhian in Southeast Asia (striped).

Dans ces différentes régions intertropicales, sans doute parce que la matière première siliceuse y est dans son ensemble peu accessible et plus rare, ce faciès est en grande partie réalisé sur de gros galets plats de forme oblongue de pétrographie diverse issus du lit des rivières (calcaire, cornéenne, grès, basalte, andésite, quartz et quartzite) (Figure 5).

La méthode de taille relève d'une activité de façonnage sur une seule face à l'aide de la technique à la percussion directe à la pierre dure mais nous verrons plus loin sur la base d'analyses faites sur des assemblages issus de fouilles récentes et bien stratifiées, que le système technique apparait plus complexe et plus diversifié qu'il n’y parait. Il repose sur plusieurs chaînes opératoires et si une première approche permet d'unifier le Hoabinhien sous le terme générique d' "industrie sur galet », une analyse plus en détail permet d'identifier sa variabilité et sa complexité.

Le Hoabinhien est présent aussi bien dans les grottes et les abris-sous-roche (Vietnam, Laos, Myanmar, Thaïlande, Cambodge) à partir d'environ 30000 ans BP, qu'associé lors de périodes plus récentes, à de vastes amas coquilliers sur les côtes, les berges de différentes 
rivières et deltas des grands fleuves d'Extrême-Orient (jusque vers 5 000-3 000 ans BP). La faune recueillie contemporaine de cette industrie est tantôt forestière, tantôt de pénéplaine voire avec des composantes marines et de mangroves pour les sites côtiers. Qu'il soit associé aux habitants des deltas, à ceux riverains des grands fleuves ou aux occupants des karsts de la zone montagneuse septentrionale de la région, cet outillage sur galet a la particularité d'être standardisé.
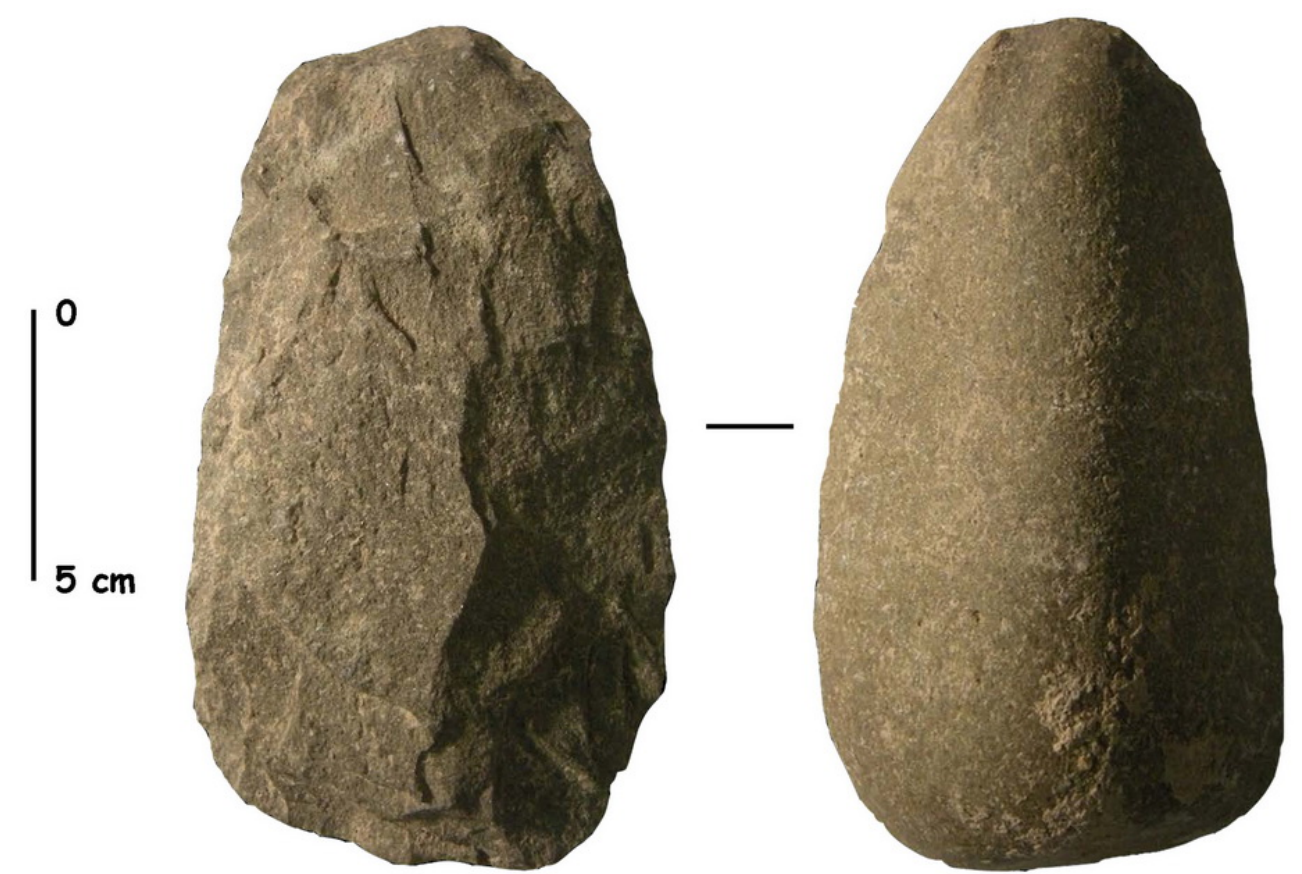

Figure 5. Exemple d'uniface hoabinhien typique (site de Laang Spean, Cambodge).

Figure 5. Typical Hoabinhian unifacial pebble (Laang Spean site, Cambodia).

L'étendue géographique du Hoabinhien est, pourrait-on dire, presque sans limite dans l'utopie diffusionniste, tant il est vrai que l'on retrouve des galets unifaciaux un peu partout hors d'Asie, en Afrique, en Amérique du Sud comme d'ailleurs en Europe de l'Ouest en France sur les terrasses quaternaires du Midi (Zeitoun et al. 2008: 45).

Si l'on tient compte de l'éclatement des limites du monde sud-asiatique et de la fragmentation des paysages, il est signalé des traces de Hoabinhien du Nord-Est de l'Inde (Hazarika 2011; Mohanty et al. 1997; Sharma 2010) jusqu'à la frange côtière sud-est de l'Australie (Bowdler 1994; 2006; Matthews 1966) voire en Papouasie (Bowdler 2006; Bulmer 1975; Groube et al. 1986). En ne ciblant que l'Asie du Sud-est, sa présence est attestée en Malaisie (Khairuddin 2010; Matthews 1960; Sieveking 1954) en passant par la Birmanie (U Aung Thaw 1971), la Thaïlande (Gorman 1969; 1970; Higham 2013; Pookajorn 1988), le Laos (Bacon 2012; Fromaget 1940; Saurin 1966), le Vietnam (Colani 1927; 1929a; 1929b; Moser 2001; Yi et al. 2008) et le Cambodge (Forestier et al. 2015; Mourer \& Mourer 1970; 1971) jusqu'à l'extrémité occidentale de l'Archipel indonésien sur l'île de Sumatra et ses petites îles (Brandt 1976; Bronson \& Glover 1984; Forestier et al. 2005a; McKinnon 1990; Van Heekeren 1972) soit de près de 2 millions de $\mathrm{km}^{2}$ (Figure 6). Concernant d'éventuelles traces sur l'île de Luzon au Philippines (Moser 2001) ou au Japon (Maringer 1957; Pearson 1976; Solheim et al. 2006: 31-32), certaines ne sont, pour le moment, pas encore confirmées ; et d'autres nécessiteraient des ré-études technologiques plus approfondies ainsi que des comparaisons avec les données des sites de nos régions d’étude. 


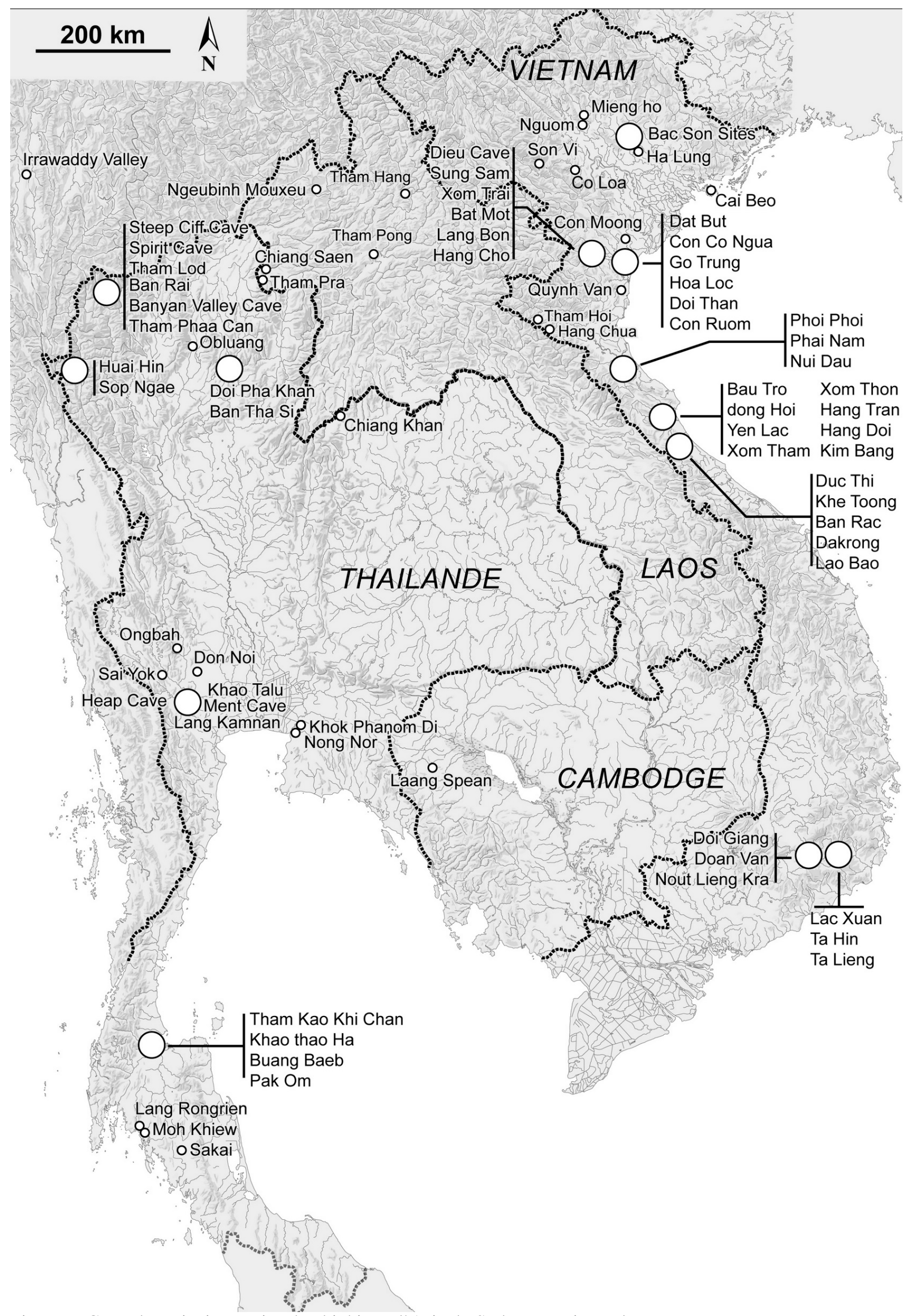

Figure 6. Carte des principaux sites hoabinhiens d'Asie du Sud-est continentale.

Figure 6. Map of the major Hoabinhian sites in Southeast Asia Mainland. 


\section{Le Hoabinhien : exception lithique de l'Extrême-Orient}

Le Hoabinhien se définit comme la culture technique traditionnelle des chasseurscueilleurs de l'Asie du Sud-est continentale que l'on retrouve aujourd'hui sur plus d'une centaine de sites (White 2011; Higham 2013). Il se présente comme la marque indélébile d'une anthropisation réussie, celle de la zone éco-géographique tropicale asiatique. En raison de sa longévité au cours des temps préhistoriques et de sa stabilité morpho-typotechnologique, l'emblématique uniface sur galet (Figure 7) confère au Hoabinhien le statut de phénomène sur plus de trente millénaires, caractérisant ainsi l'homme moderne et sa technique.
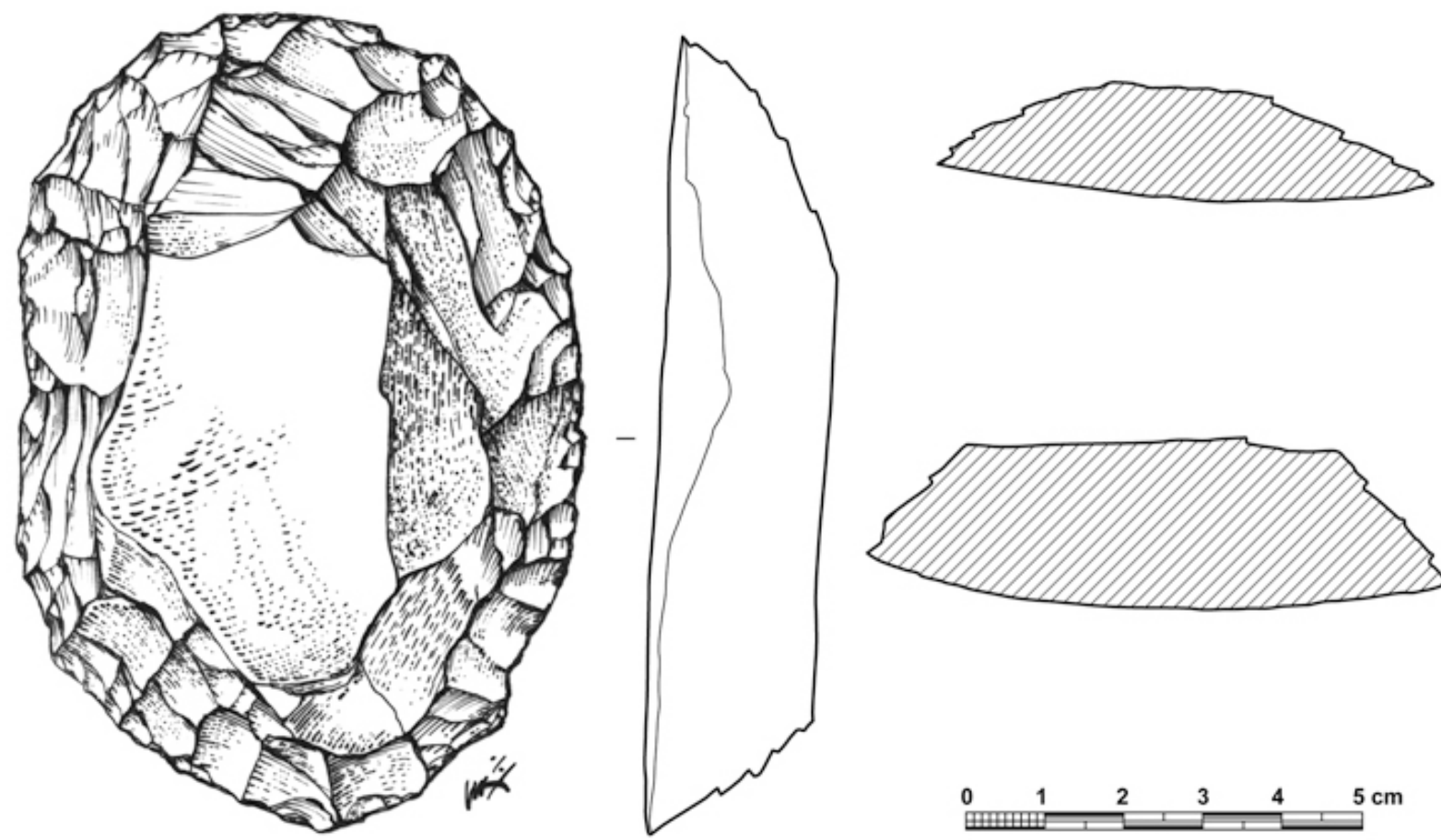

Figure 7. Uniface Hoabinhien (site de Laang Spean, Cambodge) Figure 7. Hoabinhian uniface (Laang Spean site, Cambodia).

A première vue ce phénomène pourrait traduire une résilience technique (reliquat d'archaïsmes paléolithiques) qui impose monotonie et répétition sur plusieurs dizaines de millénaires. Une stase apparente sans utilité chrono-culturelle pour les archéologues recherchant (malgré eux !) le changement, la rupture, le progrès technique ou la transition. La question préalablement posée tient à la manière dont le plus grand phénomène technique préhistorique de la zone Asie peut se résumer à de simples galets unifaciaux et sur une durée aussi longue.

Hormis l'esthétique (discutable) des unifaces (Figures 3, 5, 7 et 8), l'originalité et le caractère énigmatique du phénomène résident selon nous dans au moins trois points principaux. Le premier point correspond à la régularité et l'homogénéité des formes d'outils réalisées préférentiellement sur galet malgré la présence de matières premières siliceuses et sur une durée record de près de 30000 ans sans aucune rupture technique et autre alternative jusqu'au polissage de la pierre au Néolithique. Le deuxième point interroge la conjugaison de cette monotone pérennité d'outils massifs avec l'absence d'association d'outils lithiques pointus, à bords convergents (pointes, outils convergents bord-pointe-bord, silhouette apicale, etc.) tels qu'ils se rencontrent partout ailleurs chez les groupes de chasseurs-cueilleurs qu'ils soient du Paléolithique supérieur et subactuels. Enfin, le dernier dénonce la difficulté de placer ce phénomène technique sur l'axe évolutif des industries tel qu'il existe partout ailleurs 
dans le monde et parfois même, pour le reste de l'Asie comme en Chine, en Corée ou au Japon.
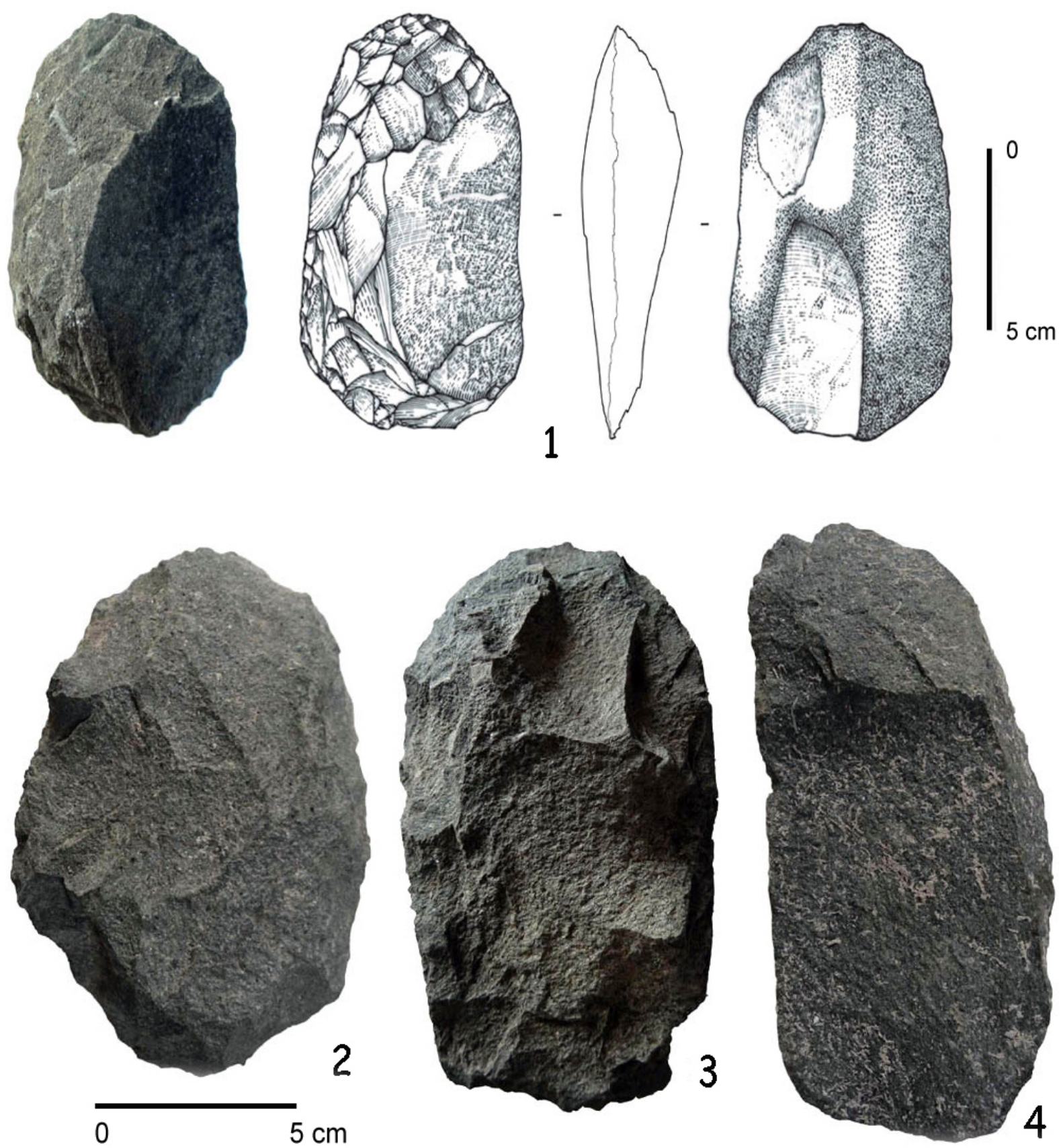

Figure 8. Diversité d’un outillage sur split de galets hoabinhiens, Laang Spean, Cambodge. 1 : chopper latéral ; 2-3 : unifaces ; 4 : outil sur split (denticulé latéral et distal).

Figure 8. Diversity of hoabinhian split pebble tools, Laang Spean (Cambodia). 1: lateral chopper; 2-3: unifaces; 4: outil sur split (denticulé latéral et distal).

Il est par ailleurs tout aussi difficile de se prononcer sur l'émergence du phénomène en termes de création "spontanée » in situ, d’innovation ou d'invention voire même de mutation depuis une lignée technique plus ancienne, comme sur sa disparition qui reste toute aussi floue. Dans ces termes, le phénomène se heurte à la thèse classique de l'évolution et du progrès technique. Autrement dit, le Hoabinhien défie le modèle classique qui, au fil du temps, converge vers la néguentropie, i.e. la complexification à la fois des données génétiques et celles relevant du progrès technique. 


\section{Les différentes facettes d'un mode original de façonnage d'outils sur galet}

C'est à partir d'études de matériel lithique de fouilles récentes et d'anciennes collections que nous avons pu expliciter les principales chaînes opératoires de façonnage sur galet de rivière réalisées selon la technique de la percussion directe à la pierre dure. Ces dernières ont été identifiées dans la quasi-totalité des sites hoabinhiens de la péninsule indochinoise notamment ceux du Cambodge, de Thaïlande, du Laos et du Vietnam.

Nos observations démontrent que la sélection précise des morphologies de galets suit plusieurs critères : une forme ovoïde épaisse ( $\geq 6 \mathrm{~cm}$ d'épaisseur) pour la confection d'outils de types chopper ou chopping-tool (qui peut aussi concerner les percuteurs), une forme allongée oblongue peu épaisse (15 $\mathrm{cm}$ de longueur en moyenne et $\leq$ à $4 \mathrm{~cm}$ d'épaisseur) de section plano-convexe utilisée pour la fabrication des unifaces; et enfin, une forme allongée épaisse et plus large $(6 \mathrm{~cm})$ de section biconvexe pour être "splité » ou " fracturé en split » (mode de percussion bipolaire sur enclume) et ainsi obtenir deux hémi-galets.

Ces trois types de support-galet renvoient à trois chaînes opératoires bien individualisées utilisant chacune un schéma de façonnage répondant à une construction volumétrique particulière selon des critères techniques propres à l'activité de taille sur galet (Forestier et al. 2005a; 2005b; 2013; Zeitoun et al. 2008). Seule la troisième chaîne opératoire fait intervenir deux schémas opératoires imbriqués: l'un de façonnage et l'autre de débitage ce qui lui confère une relative complexité et longueur en terme d'étapes. Ce schéma est alors dit mixte (débitage + façonnage) et a pour objectif de produire des split de galet ou « fracturation en split » (Faivre et al. 2010).

L'étape de prédétermination du façonnage hoabinhien est guidée par la sélection préalable de formes particulières de galets (de section plano-convexe et bi-convexe ou ovoïde) impliquant des volumes précis aux pièces unifaciales de facto standardisées, chose rare dans les industries sur galets d'époque plus ancienne du Quaternaire (Forestier et al. 2014; Pope et al. 1987; Zeitoun et al. 2012b).

Les trois chaînes opératoires rencontrées reposent sur un schéma de façonnage du plus simple au plus complexe :

- Une chaîne opératoire courte : réalisée à partir de galets ovoïdes épais ( $\geq 6 \mathrm{~cm}$ d'épaisseur) afin de dégager un fil tranchant de types chopper (Figure 9) ou chopping-tool. Il s'agit d'un façonnage effectué par une série limitée d'enlèvements en partie distale (entre 3 et 5 éclats).

- Une chaîne opératoire médiane (plus longue que la précédente) : correspond au façonnage d'unifaces sur galet de forme allongée, oblongue peu épais (15 cm de longueur en moyenne et $\leq$ à $4 \mathrm{~cm}$ d'épaisseur) et de section strictement plano-convexe. La morphologie naturelle plano-convexe du galet délimite un plan d'intersection ainsi qu'une hiérarchisation entre surface de plan de frappe (plan ou cortical) et surface façonnée (convexe ou bombée) (Figure 10). Ainsi réalisé, le façonnage d'un uniface suit un épannelage partiel ou périphérique, autrefois appelé non sans humour par F. Bordes «biface de fainéant » (1961) ou « biface de malin » (1974).

La configuration volumétrique propre à cette modalité de façonnage est plus simple car imposée par la forme naturelle du galet sélectionné c'est-à-dire au plus proche de l'outil souhaité. La morphologie d'origine est alors maintenue quelque soit l'avancée du façonnage et reste donc inchangée sur un plan morpho-technologique durant le temps de la chaîne opératoire. Cette chaîne opératoire de façonnage comme d'ailleurs les deux autres ne compte pas d'étape stricto sensu de phase de mise en forme ou préforme car la construction volumétrique est imposée par la morphologie naturelle et structurelle (plano-convexe) des galets sélectionnés dans les rivières. 


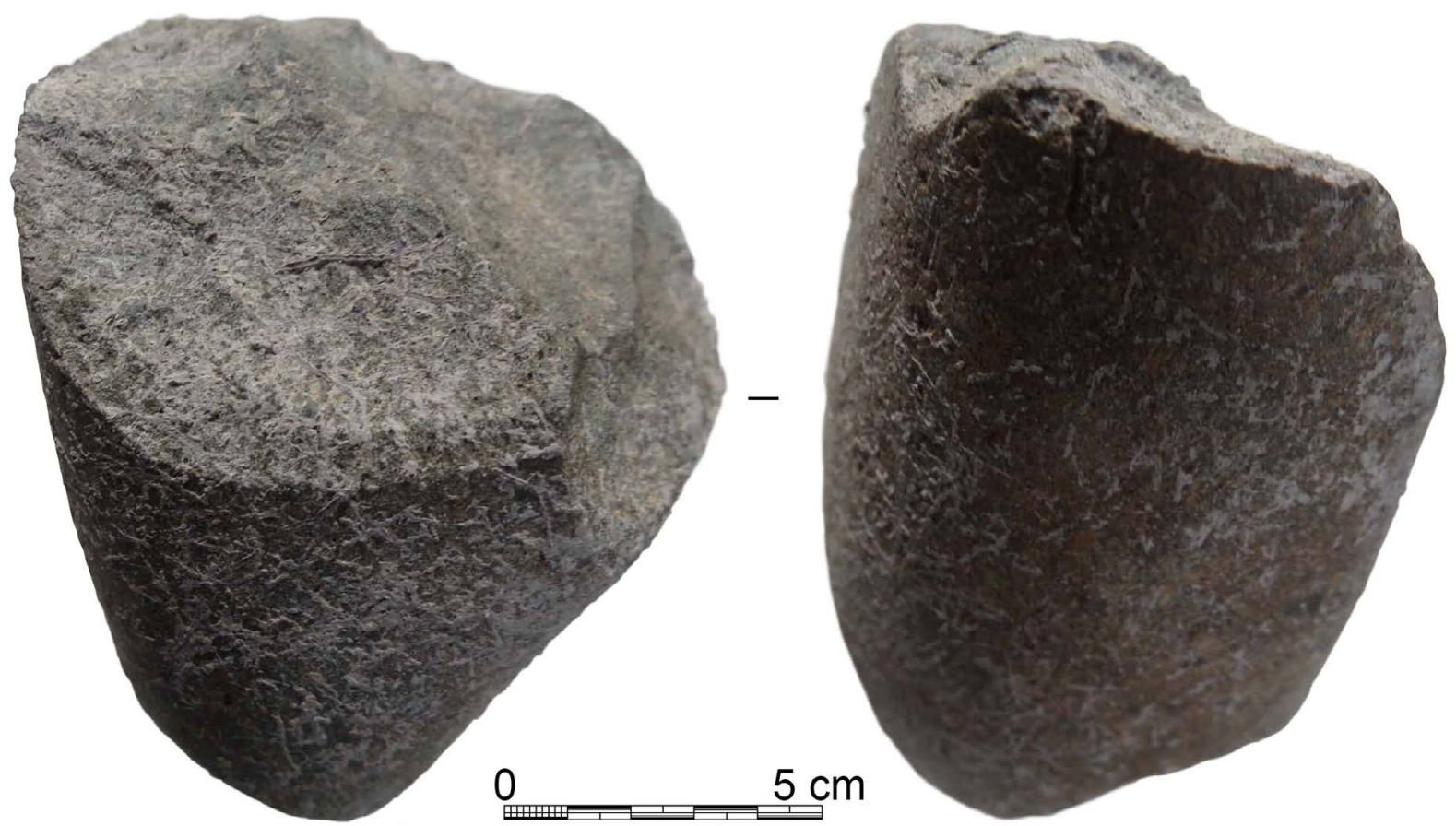

Figure 9. Chopper, site en grotte hoabinhien de Laang Spean (Cambodge).

Figure 9. Chopper, Hoabinhian site of Laang Spean cave (Cambodia).
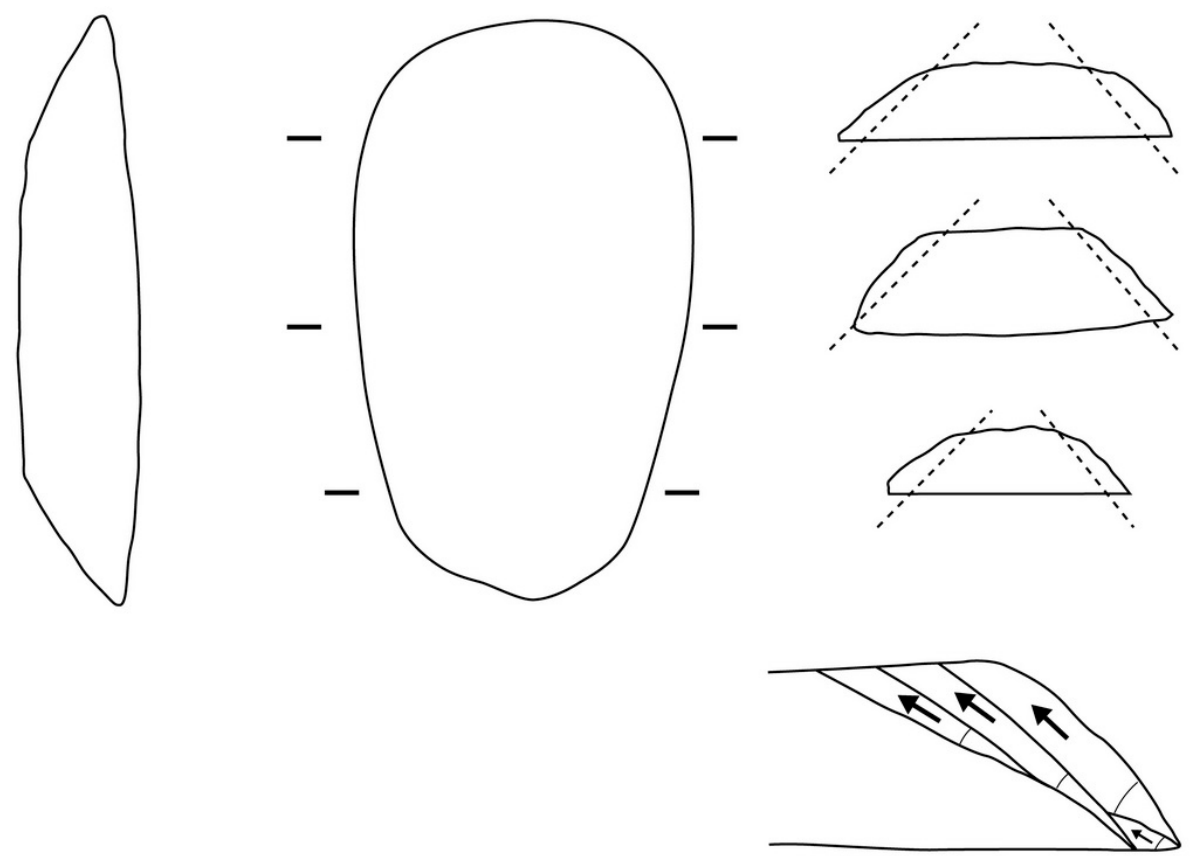

Figure 10. Schéma synthétique d’aménagement d’un galet de section plano-convexe en partant de la surface plane vers la surface convexe.

Figure 10. Schematic drawing of a plano-convex pebble from the flat surface to the convex surface.

En revanche, il existe une hiérarchisation des sous-produits dans l'amincissement du galet avec au moins trois séries principales d’éclats prédéterminés et pré-déterminants. Leurs caractéristiques morpho-technologiques ont été individualisées en plusieurs catégories : éclats corticaux (entames) trapézoïdales plus larges que longs, éclats peu ou pas corticaux quadrangulaires-allongés (avec négatifs d'enlèvements unidirectionnels) et éclats dits en « aile d'oiseau » (Figure 11). Ces trois séries d'éclats constituent les étapes du façonnage, 
répétées selon l’ordre chronologique autant que technologique en $1^{\text {ère }}, 2^{\text {ème }}$ et $3^{\text {ème }}$ série (génération) sur toute la périphérie du galet support jusqu'à l'obtention du fil tranchant ou de la spécificité fonctionnelle du tranchant voulue et recherchée par le tailleur. Dans sa «vision artisanale de l'outil » comme il l'a nommé, M. Lepot (1993) parle d' « angle de coupant » et de " plan de coupe » formé par la convergence de deux surfaces dégageant un dièdre de coupe aménagé pour l'instrumentation.

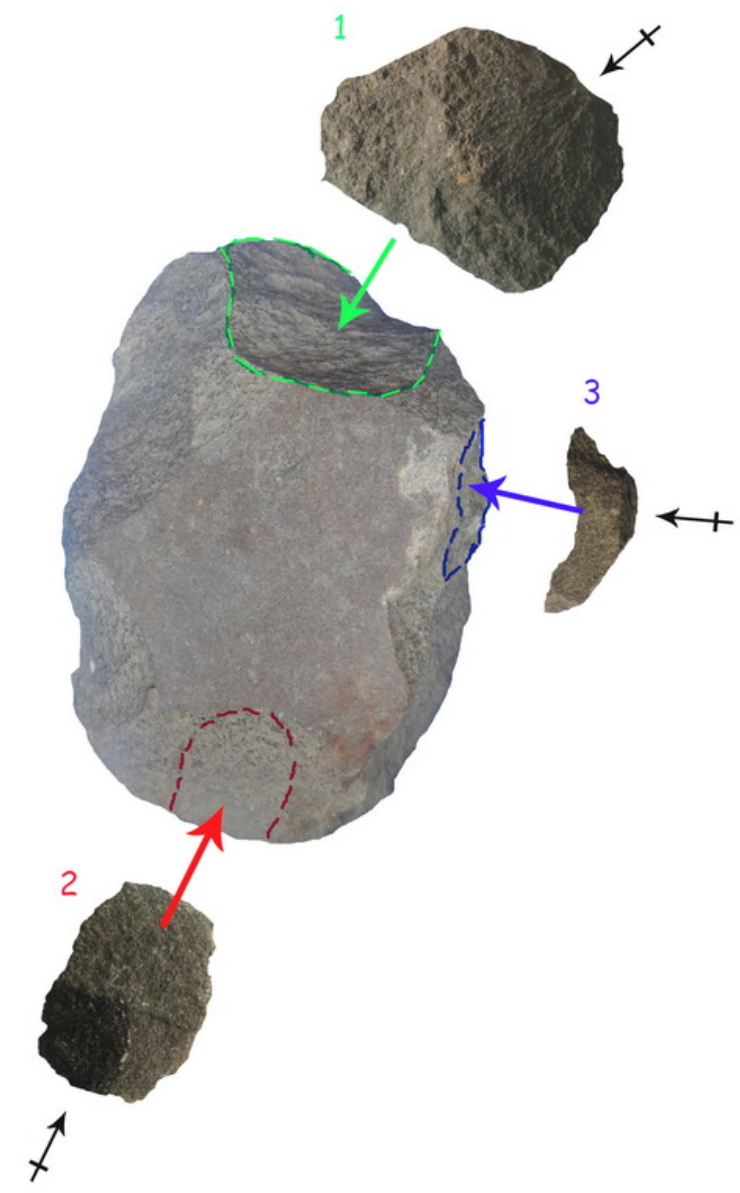

1ère série d'éclats corticaux plus larges que longs, à contour trapézoïdal et à profil convexe. Premiers enlèvements de façonnage profonds et semi-abrupts.

3éme série d'éclats marque la fin du façonnage et la confection d'un angle tranchant. Ces éclats plus larges que longs en "ailes d'oiseau" laissent des négatifs d'enlèvements semi-abrupts et abrupts qui aménagent un angle de coupant (bord actif) compris entre $40^{\circ}$ et $60^{\circ}$.

2éme série d'éclats de façonnage, peu ou pas corticaux, à contour quadrangulaire allongé ou rectangulaire. Ces éclats laissent des négatifs d'enlèvements généralement abrupts ou obliques.
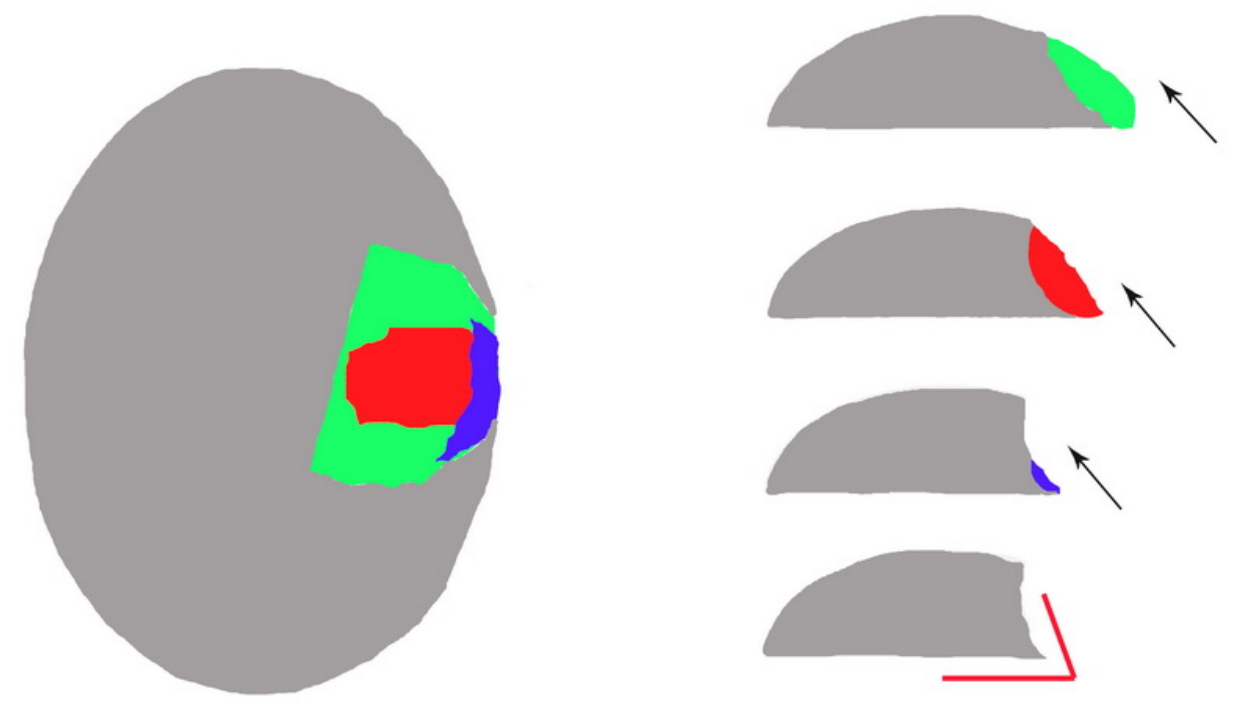

1 ère série

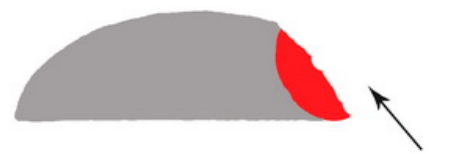

2ème série

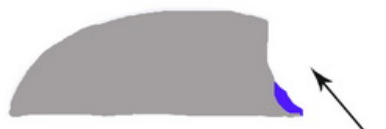

3ème série

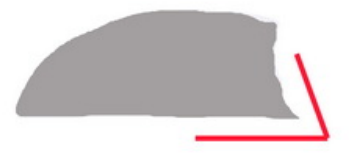

Aménagement de l'angle de coupant

Figure 11. Chaîne opératoire de façonnage d'un uniface hoabinhien.

Figure 11. Chaîne opératoire of Hoabinhian uniface shaping. 
Comme dans le cas du façonnage du biface, l'uniface présente des caractéristiques morpho-techniques particulières liées à une sélection de galets plats et oblongs de section plano-convexe présents dans l'environnement. Ce qui implique des modalités à la fois «autostructurantes » et «initialisantes » dès le ramassage avec une orientation logique du façonnage vers la surface corticale la plus naturellement convexe (la plus bombée $=$ réservoir de matière). Dans ce cas, s'opère alors la projection d'une asymétrie "virtuelle », i.e. l'image mentale du produit hiérarchisant surface de plan de frappe (surface plane) ou surface façonnée (convexe). La recherche d'un plan d'équilibre unifacial c'est-à-dire, le contrôle d'une asymétrie constante entre la face naturelle (plane) et la face exploitée (convexe) ainsi que le caractère prédéterminé d'une base naturelle corticale (plane) à partir de laquelle s'organise le façonnage $\left(1^{\text {ère }}, 2^{\text {ème }}\right.$ et $3^{\text {ème }}$ série d'éclat), impliquent un fort degré de prédétermination de ladite surface.

Cette chaîne de façonnage obéit à un principe dialogique simple reposant sur le maintien d'un déséquilibre structurel induit par l'asymétrie de deux surfaces: l'une plane, l'autre convexe. L'asymétrie plano-convexe héritée de la forme naturelle du galet sélectionné, s'affiche comme une des spécificités du façonnage unifacial sur galet plat: la signature technique du Hoabinhien. Cette dernière s'organise en au moins quatre épisodes structurants notés I, II, III et IV (du plus clair au plus foncé dans la Figure 12) qui en assurent l'auto-écoorganisation. La morphologie d'une pièce unifaciale plano-convexe peut se modifier progressivement à la suite d'épisodes de retouches successives des bords (Figures 10, 11 et 12).

Ainsi, lorsque sont atteints les épisodes III ou IV, on observe une exploitation quasitotale du galet ce qui confère une instrumentalisation maximale du pourtour de la pièce. La lecture d'un uniface nous montre que le galet tel qu'il est voulu, est façonné en trois à quatre épisodes incluant trois séries d'enlèvements (techno-types) qui ont pour but d'aménager un fil tranchant tout en gardant la section plano-convexe à savoir, les premiers enlèvements de mise en forme du volume (épannelage, premiers gestes de façonnage), les enlèvements de préaffutage et enfin, les petits enlèvements d'affûtage qui individualisent des " unités technofonctionnelles » ou UTF (Boëda 2001; 2013; Lepot 1993). Ces dernières renvoient précisément au fonctionnement global de l'outil par la reconnaissante des parties actives et préhensives selon une lecture dite «morpho-techno-fonctionnelle » (Boëda 2013).

Pour l'heure, d'un point de vue strictement typologique il nous est difficile de distinguer des types particuliers d'outils dans la grande famille des galets unifaciaux. On ne peut qu'observer des types illimités de formes d'outils sur galet plat avec $n$ tranchants actifs. Tous sont de section plano-convexe et difficilement distinguables les uns des autres car répondant à la même construction volumétrique, allant de pièces ovalaires jusqu'aux limandes auxquelles il faut rajouter l'attribut de partiel, de total ou latéral à l'étendue du façonnage et du bord retouché (Figures 7 et 13).

La difficulté de nommer ce type d'industrie n'est pas propre au monde asiatique, elle a été perçue très tôt pour les séries du Paléolithique archaïque européen et en particulier pour les outils à tranchant dit «terminal " (type chopper). Mais aussi pour l'ensemble du groupe des unifaces dit «à dos » (Le Brun-Ricalens 1989), " chopper latéral à tranchant étendu » (Collina-Girard 1986) ou encore « unifaces à tranchant distal convexe » (Tavoso 1978).

Nommer précisément ces pièces à partir de critères strictement morphologiques serait vain, seule une « approche techno-morpho-fonctionnelle » (Boëda 2001) pourrait, selon nous, apporter un plus à la classification de ces formes d'outillage sur galet. En effet, la variabilité et la nature de la retouche comme de l'étendue et la qualité des tranchants sur la totalité ou pas de la périphérie de chaque pièce, pourraient permettre d'avancer des critères distinctifs entre un type d'outil et un autre sur la base de la reconnaissance des unités technofonctionnelles (UTF). Comme d'ailleurs beaucoup d'outillages sur galet du Paléolithique 
ancien, l'outillage hoabinhien répond à un certain nombre de règles propres, régies par une logique organisée autour de l’asymétrie plano-convexe.
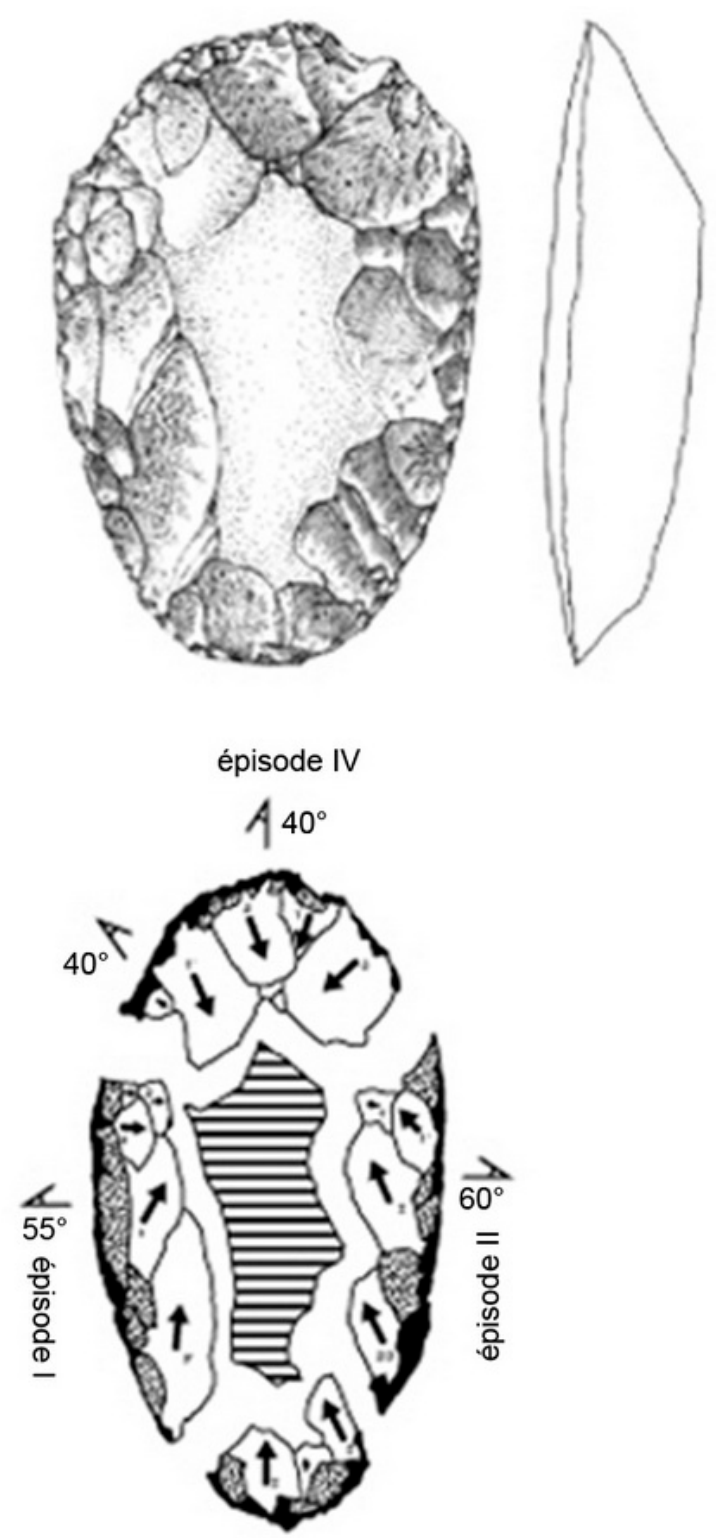

épisode II

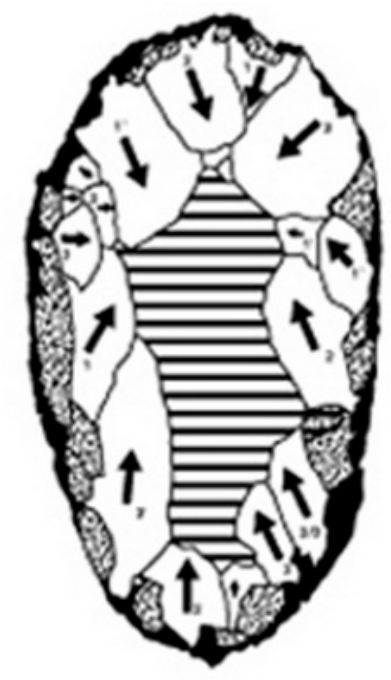

Épisodes structurants I, II, III, IV Générations d'enllèvements (par ordre chronologique : du plus chair au plus foncé)

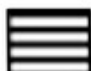
réservoir de matière (convexité naturelle) $\square$ Construction du volume (épannelage) pré-affûtage affûtage (point de contact transformatif)

Figure 12. Exemple d'analyse diacritique d'un uniface, site Thank Khoa, Nord Vietnam (coll. Colani, Musée de l'Homme, Paris) (modifié d'après Forestier 2000).

Figure 12. Example of diacritical analysis of a uniface, Thank Khoa, North Vietnam (Colani coll., Musée de l’Homme, Paris) (modify after Forestier 2000).

- Une chaîne opératoire longue et mixte à la fois : un degré supplémentaire vers la complexité. Il s'agit de l'imbrication d'une activité de débitage et de façonnage. Cette chaîne mixte est appliquée sur des galets oblongs de section biconvexe, en moyenne plus épais que ceux sélectionnés pour les deux autres chaînes opératoires $(6 \mathrm{~cm})$. L’objectif est d'atteindre les caractéristiques morpho-techniques spécifiques à l'uniface. Pour cela, le galet doit être préalablement réduit en volume ou, pourrait-on dire, «initialisé » par une étape d'amincissement. Le galet est alors «splité » c'est-à-dire fracturé en deux parties plus ou moins égales soit deux hémi-galets : l'un est dit positif (split A avec contre bulbe), l'autre est dit négatif (split B sans contre bulbe mais avec esquilles et arrachements). Comme le montre 
le schéma opératoire de la Figure 14 : le split est produit soit longitudinalement c'est-à-dire dans l'axe morphologique naturel du galet soit transversalement (à partir d'un des côtés). Cette opération se fait au moyen d'une technique à la percussion directe à la pierre dure ou bien, selon un mode de percussion bipolaire sur enclume (surtout dans le cas des split longitudinaux).
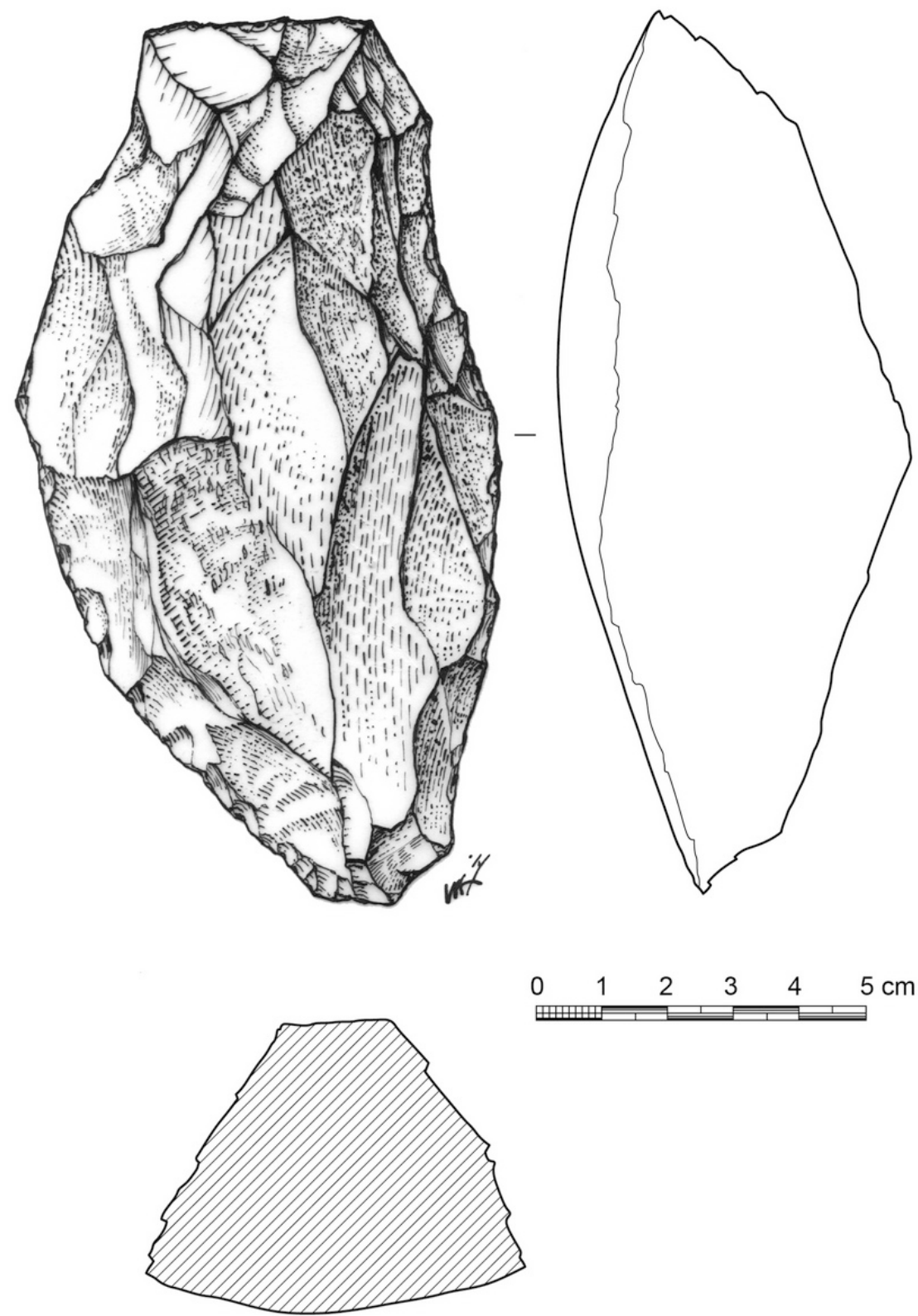

Figure 13. Uniface, Laang Spean (Cambodge).

Figure 13. Uniface of Laang Spean, Cambodia.

L'obtention des splits (A/B) permet de retrouver une morphologie plano-convexe et donc une asymétrie des surfaces qui sera ensuite confectionnée au moyen d'épisodes structurants (retouches) signalés précédemment (notés I à IV) pour atteindre les mêmes objectifs morphofonctionnels que la chaîne opératoire de l'uniface. Lors de la phase de confection de l'outil, la retouche est alors orientée vers la face d'arrachement convexe ou plane non corticale. La confection du tranchant peut être partiellement lue sur le split-outil, lorsque l'aménagement par la retouche n'affecte pas l'entière périphérie du pourtour de la zone du bulbe ou du contrebulbe laissée libre. 


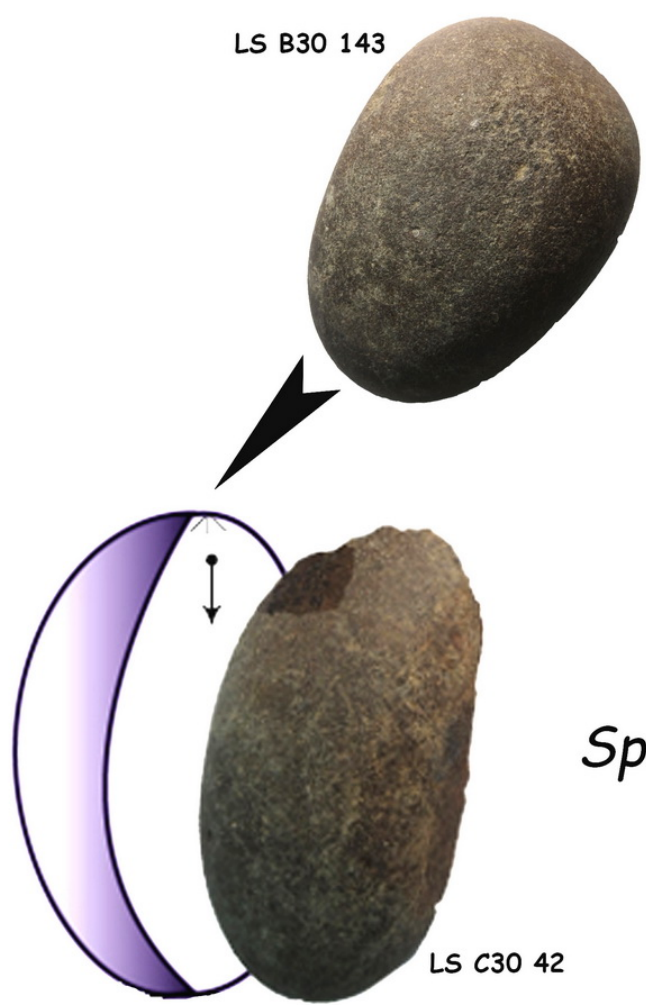

Split longitudinal

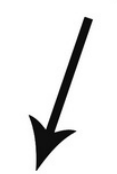

Split A

Base positive Base négative

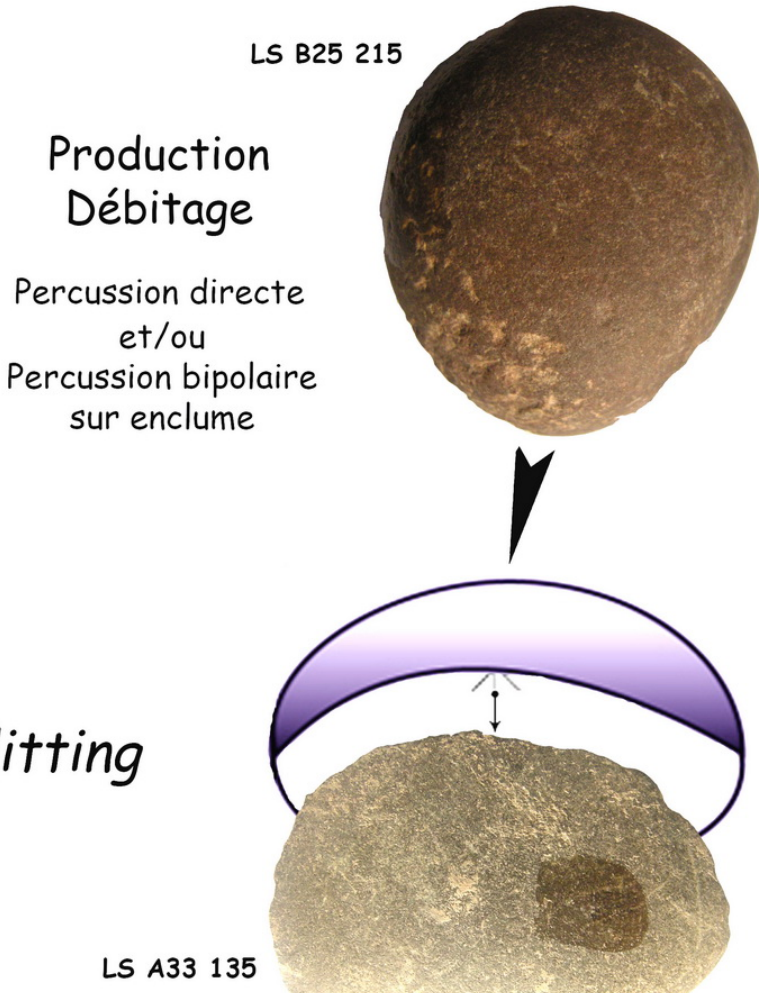

Split transversal

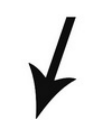

Split A

Base positive $\downarrow$

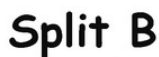

Split B Base négative

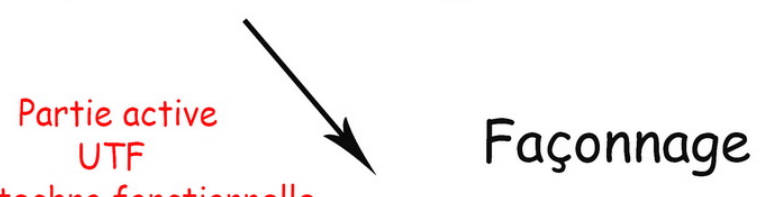

unité techno fonctionnelle

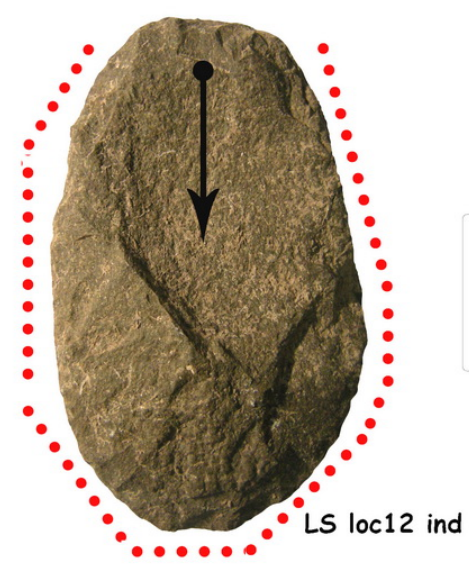

Phase de confection de la partie tranchante (active)

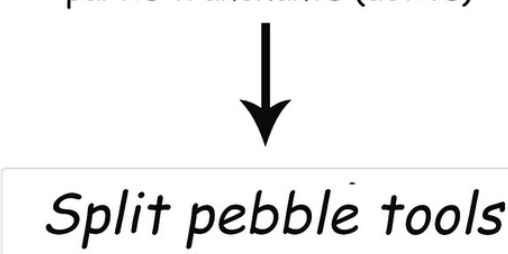

Outils sur split de galet

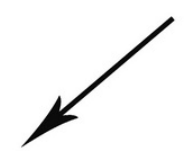

Partie active

UTF

unité techno fonctionnelle

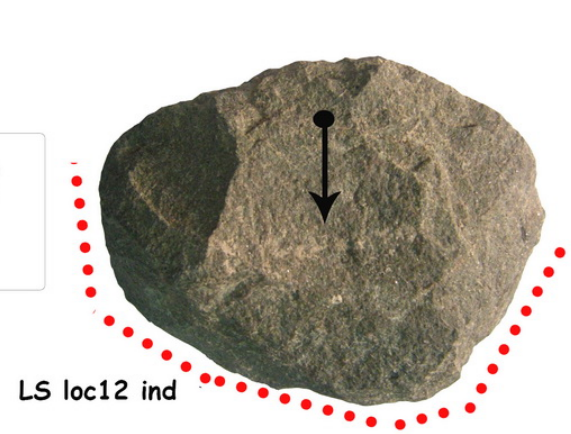

Figure 14. Schéma synthétique des différentes étapes dans la fracturation en split d'un galet.

Figure 14. Synthetic sketch of the different steps of a split pebble fracturation. 
Cette méthode dite «mixte » faisant intervenir un débitage-fracturation suivi d'un façonnage (confection de l'outil), interroge l'identité même d'un galet «support d'outils multiples ». Dans ce cas précis, il pourrait être interprété non pas comme une matrice à façonner mais comme une sorte de «nucléus » générateur de supports ensuite aménagés en outils divers (racloir convexe-droit-concave, coche, denticulé) ou utilisés brut de taille.

Cette chaîne opératoire est sans doute la plus complexe des trois mais aussi, la plus évolutive car elle repose sur une double configuration volumétrique du galet ouvrant à une plus grande variabilité des formes d'outils sur split (Figures 15 et 8), plus légers que les unifaces avec parfois des formes pointues (Figure 16).

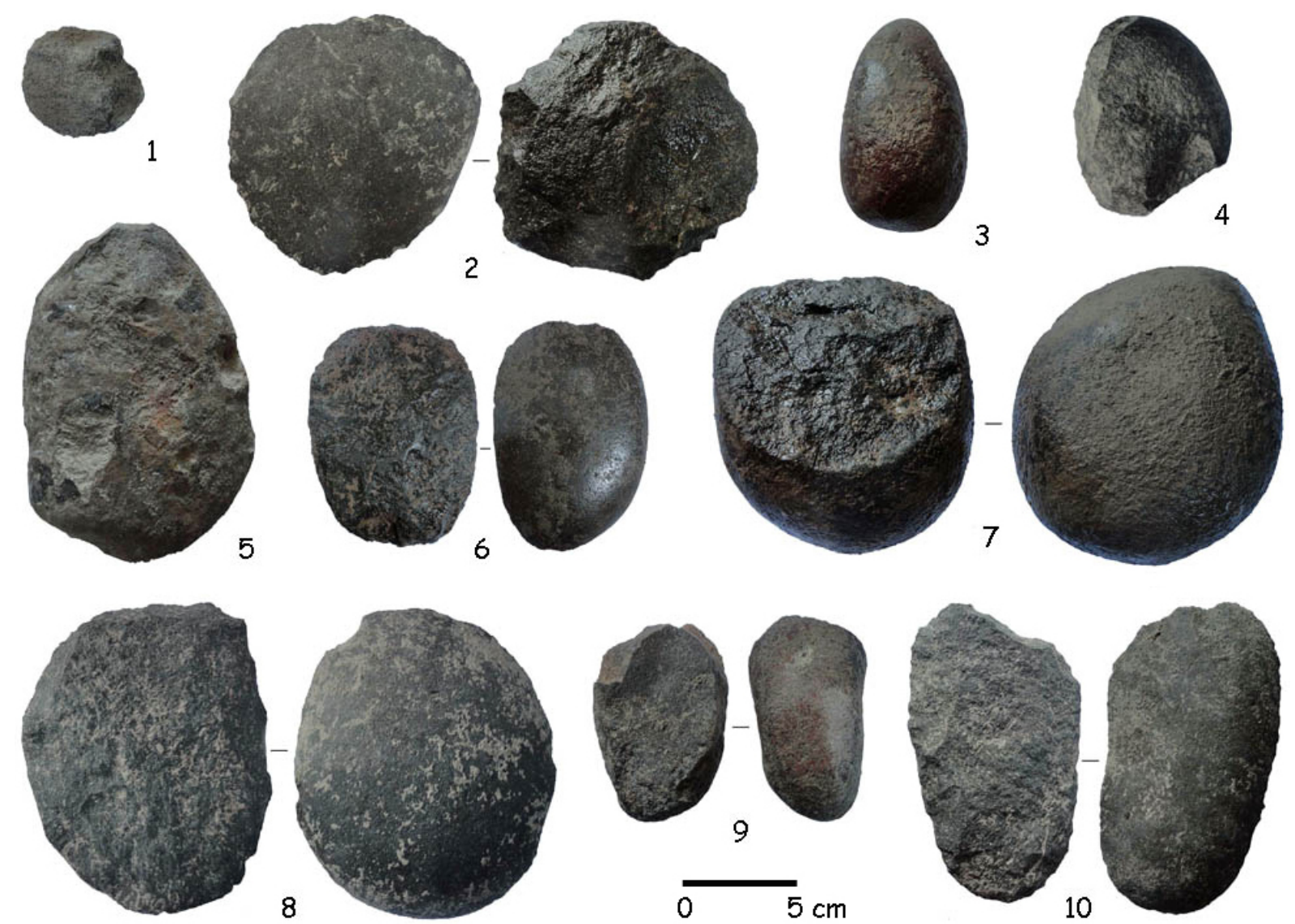

Figure 15. Diversité d'un assemblage hoabinhien, Laang Spean, Cambodge (split, non split). 1-7 : non split ; 810 : split.

Figure 15. Diversity of hoabinhian assemblage, Laang Spean (Cambodia) (split, no split). 1-7: no split; 8-10: split.

Sa complexité comme son efficacité se traduisent aussi sur un plan économique. A partir d'un galet elle produit deux splits qui à leur tour vont porter sur leur périphérie une série de tranchants : un support à tranchants multiples. Dans ce cas particulier, on peut dire aussi que nous nous trouvons devant un double concept économique réunissant d'une part, une économie de «l'outillage " (Inizan 1976) et d'autre part, une économie de «la matière première » (Perlès 1980).

Ainsi, le Hoabinhien tel que nous l'avons observé depuis plus d'une décennie (Forestier 2000; Forestier et al. 2005b; 2015) dans de nombreux sites d'Asie du Sud-est repose sur au moins ces trois chaînes opératoires distinctes et complémentaires dans un même assemblage (Figure 17). 

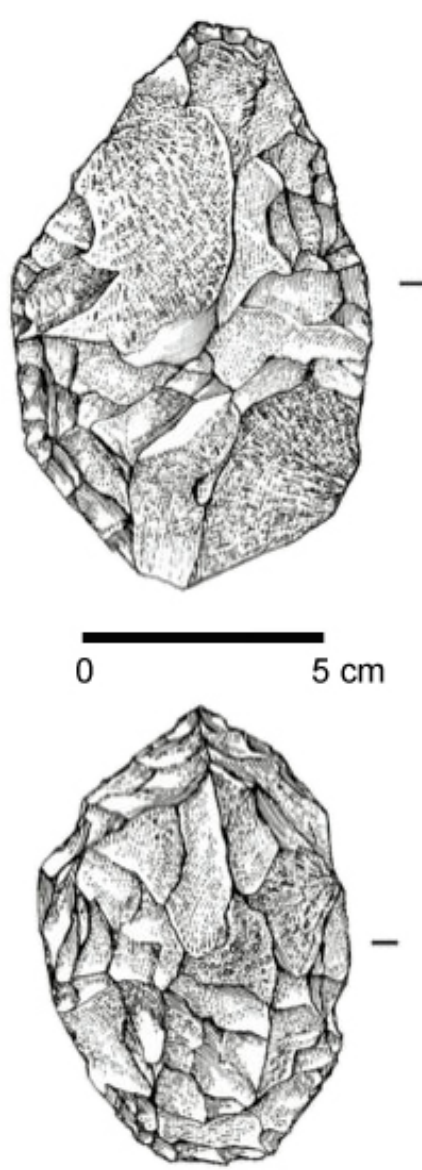
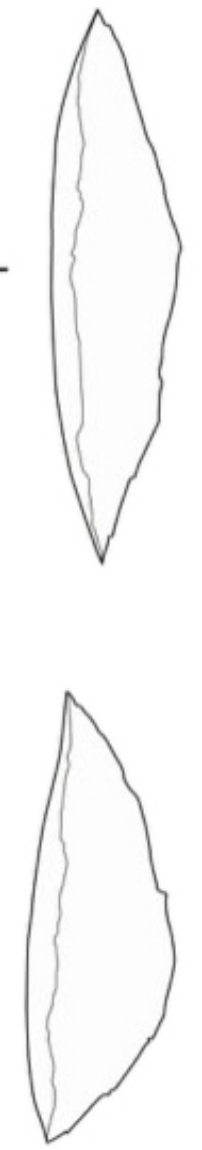

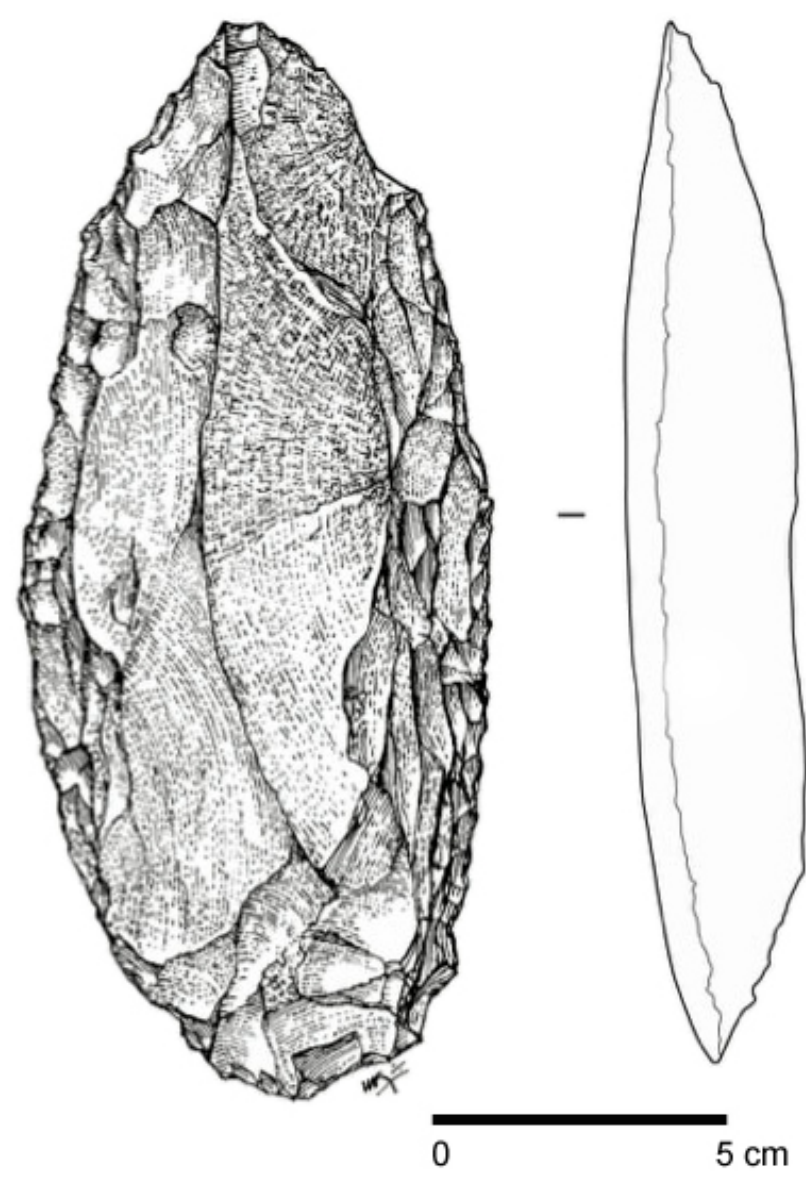

0

Figure 16. Unifaces réalisé sur split, Luang Prabang, Mékong (Laos).

Figure 16. Uniface made on split from Luang Prabang, Mekong river, (Laos).

Le site de Laang Spean au Cambodge refouillé depuis 2009 par la Mission Préhistorique Franco-Cambodgienne (Forestier et al. 2015; Sophady et al. 2015) montre très nettement la forte représentation des outils sur split (50\% des outils sur galet) par rapport aux unifaces (classiques, 19\%) et aux chopper ou chopping tool (31\%) réservés à des galets plutôt épais et ovoïdes.

Ces trois chaînes qu'elles soient longues, courtes ou mixtes sont toutes sous-tendues par une activité dominante de façonnage. Complémentaires et originales, elles ont pour but d'obtenir des outils variés sur galet, à la fois des macro-outils (uniface, chopper, outils sur split, etc.) mais aussi, un petit outillage réalisé sur les éclats de façonnage plutôt épais et plus larges que longs ( $2^{\text {ème }}$ et $3^{\text {ème }}$ générations). Comme cela avait déjà été avancé pour la Thaïlande (Forestier et al. 2013) et plus récemment pour le Cambodge (Forestier et al. 2015), l'analyse d'un micro-outillage sur éclats parmi un ensemble d'outils plus massifs est à reconsidérer sur un plan statistique et techno-morpho-fonctionnel ou tracéologique. Ces outils sur éclats (micro-outillage) peuvent être classés en outils bruts de taille non retouchés ou en racloir, micro- denticulé, coche pour les plus représentatifs et plus rarement en bec (Figure 18). 


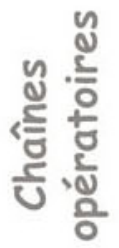

Uniface

흔

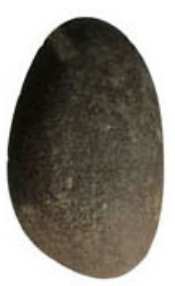

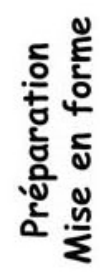

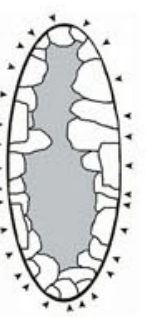

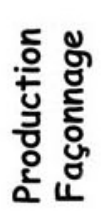

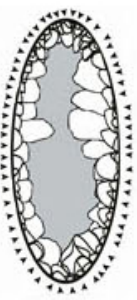

כo

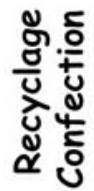

Eclats de façonnage retouchés ou utilisés bruts
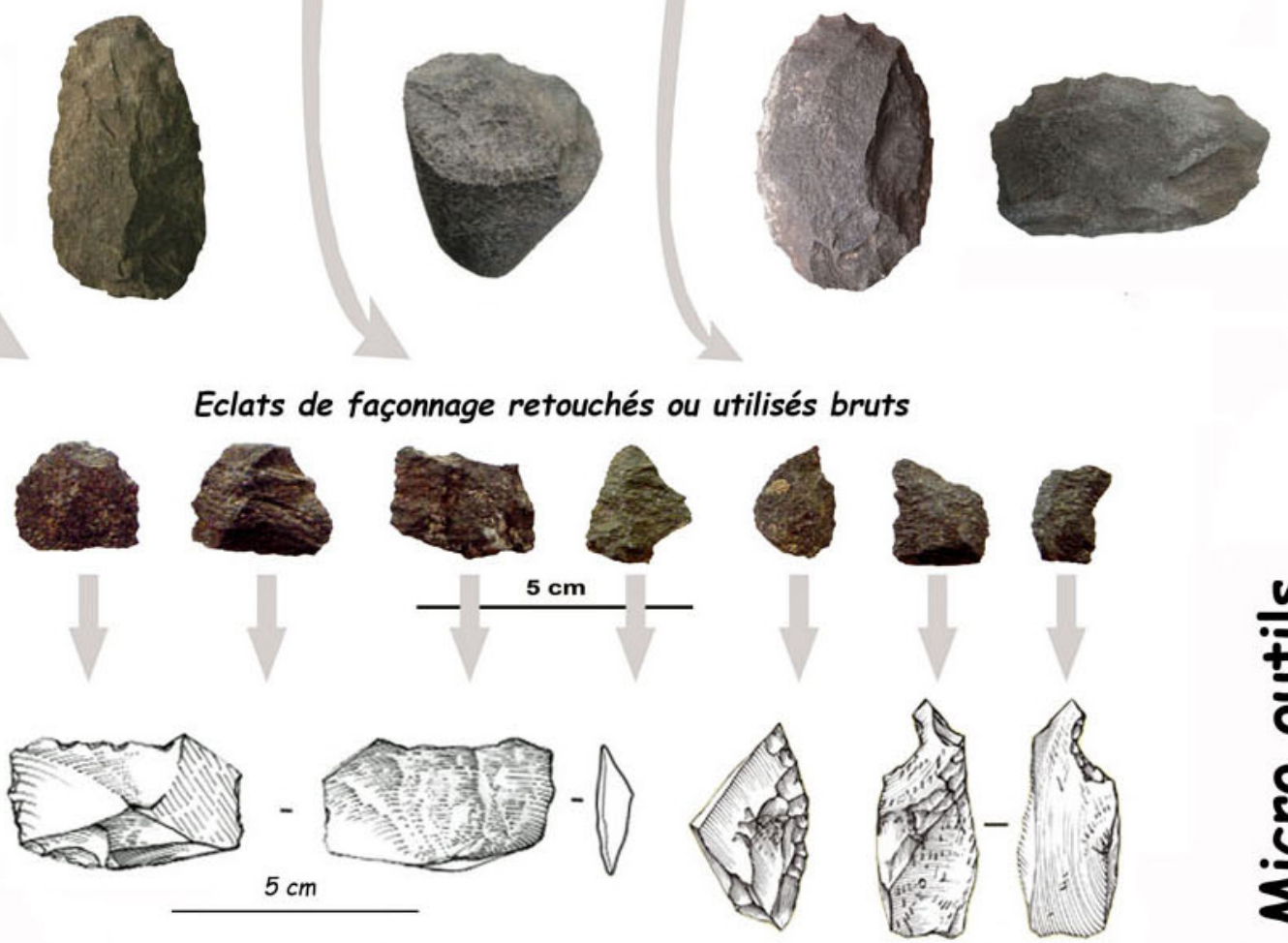

$\frac{n}{5}$
$\frac{5}{0}$
0
$\frac{2}{2}$
$\frac{2}{3}$

Micro outils sur éclat de façonnage (1ère et 2ème série) (racloirs, denticulés, encoches, becs)

Figure 17. Chaines opératoire sur galet, assemblage hoabinhien, Laang Spean (Cambodge).

Figure 17. Chaîne opératoire on pebble assemblage, Hoabinhian, Laang Spean (Cambodia). 

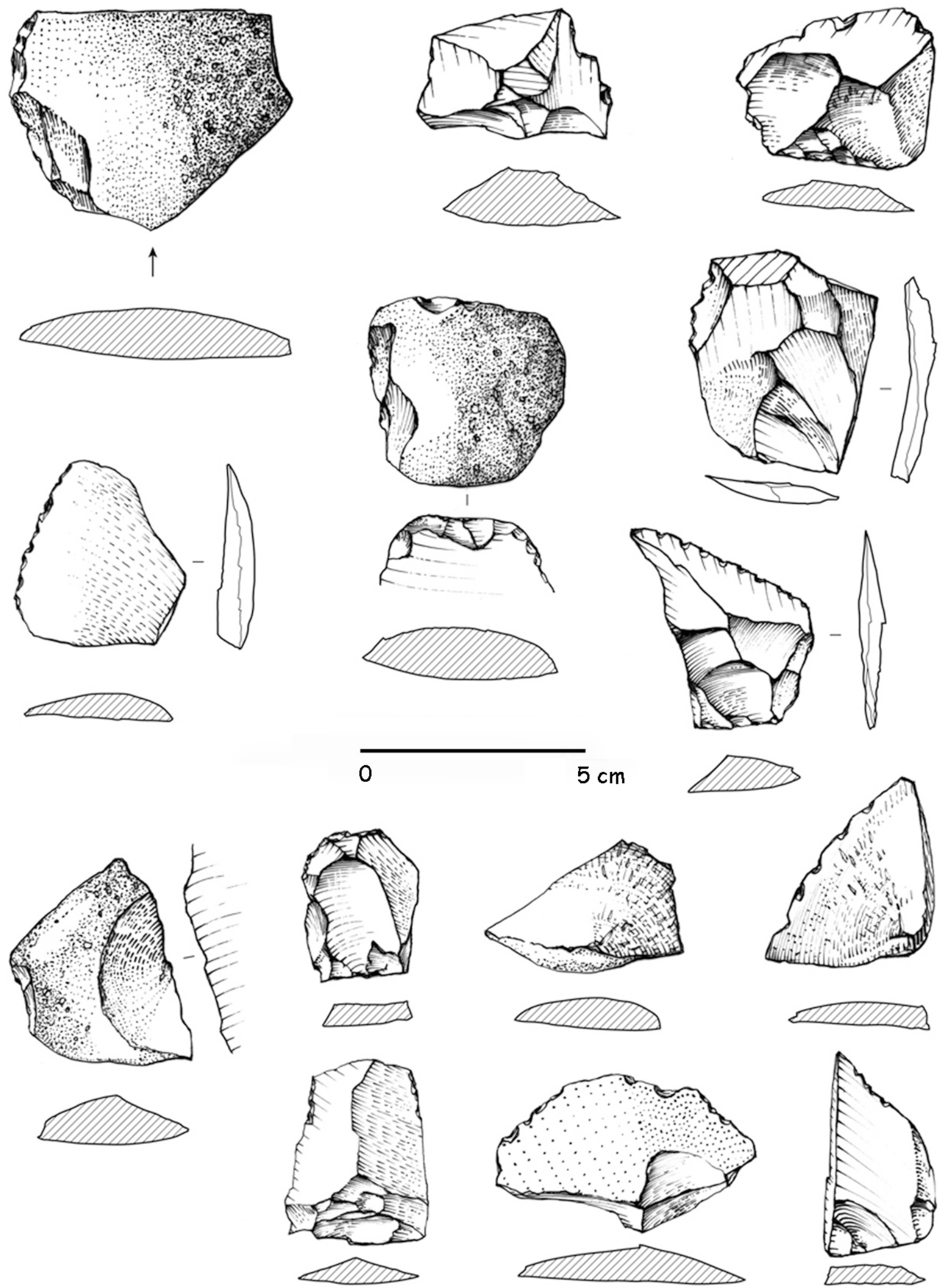

Figure 18. Petit outillage sur éclats de façonnage d'uniface.

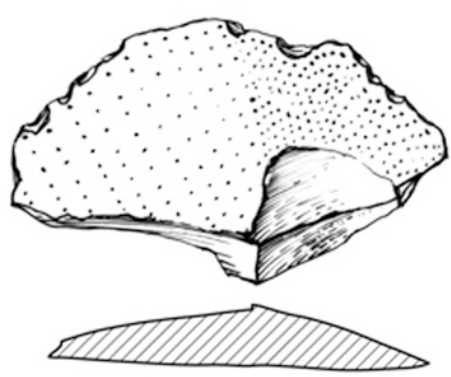

Figure 18. Small flake tools from unifacial shaping. 


\section{Le Paléolithique final du Sud-est asiatique : multiplicité et inertie du Hoabinhien}

De récentes découvertes nous poussent à penser que le Hoabinhien serait né non pas au Vietnam, lieu historique de son expression et de son expansion mais, en Chine du Sud dans le Yunnan où il aurait été très probablement testé, inventé non loin des berges du Fleuve Mékong. Les récents résultats obtenus dans l'abri-sous-roche Xiaodong dans la province du Yunnan donnent une date de 43000 ans BP pour son éclosion, ce qui permet d'avancer l'hypothèse logique d'un «Hoabinhian Homeland " (suggérée par notre équipe) en Chine du Sud où le Mékong entame sa course transfrontalière de près de $2000 \mathrm{~km}$ pour atteindre son delta au Sud Vietnam et changer son nom en "fleuve des neuf dragons " (Xueping et al. 2016).

L’origine du Hoabinhien doit être à présent reconsidérée sur la base de nouvelles recherches (en cours) menées dans les territoires chinois frontaliers de la Chine du Sud comme les provinces du Yunnan, du Guangxi et du Guangdong. Quant à sa manifestation la plus récente, elle est proche de 3700 BP en Thaïlande du Nord ce qui implicitement remet aussi en question l'apparition et la nature du Néolithique stricto sensu en Asie du Sud-est continentale (Forestier et al. 2013; Zeitoun et al. 2008).

Comment dessiner une vue d'ensemble de ce monde technique ?

Le Hoabinhien peut être vu comme une invention technique provenant du Sud de la Chine pour ainsi dire, une idée technique «nomade » trans-Asie dont l'originalité se reflète dans une certaine conception de la surface tranchante du galet.

De cette innovation s'ensuivent des modifications et ajustements peut-être même amélioration(s) aboutissant à une variabilité et à une maturité technique (méthodes et outillages) en Asie du Sud-est. Ceci pouvant expliquer sa stabilité temporelle, structurelle, fonctionnelle autant que spatiale sur près de 2 millions de $\mathrm{km}^{2}$ pendant trente millénaires, preuve d'une adaptation et d'un peuplement réussis dans un environnement forestier intertropical compris entre le $25^{\circ} \mathrm{N}$ et le $5^{\circ} \mathrm{S}$ environ.

Les études régionales consacrées aux paléoclimats pléistocènes et holocènes de la péninsule Indochinoise (Maxwell 2001; Penny 2001; 2006) montrent le passage d'un climat plus froid et plus sec qu'à l'actuel à des conditions beaucoup plus humides à la limite du Pléistocène et de l'Holocène (12 000-10 000 ans BP). De toute évidence ces nouvelles conditions (moussons) permettent l'expansion des taxons tropicaux et le développement d'une végétation comparable à la flore moderne jusqu'à 6000 ans BP. C'est dans ce contexte environnemental que s’est développé, pérennisé et épanoui le Hoabinhien.

Au delà des premières interrogations (Reynolds, 1990), les nouvelles données issues des fouilles récentes de niveaux hoabinhiens dans les principaux pays d'Asie du Sud-est (Moser 2001; Rabett 2012; Zeitoun et al. 2008) comme en Thaïlande (Auetrakulvit et al. 2012; Marwick 2007; 2008a; Schoocongdej 2006), au Cambodge (Forestier et al. 2012; Sophady 2014; Sophady et al. 2015), au Laos (Bacon 2012; White et al. 2009; Zeitoun et al., 2012a), au Vietnam (Rabett et al. 2009; Yi et al. 2008) permettent aujourd'hui de réfléchir sur un élargissement de la notion de Hoabinhien non pas tant sur un plan spatial mais sur son statut de technocomplexe en coordination avec une révision générale de son tool-kit et de ses chaînes opératoires. D’ailleurs, les approches technologiques (reconnaissance des méthodes et des techniques de taille) avec l'utilisation du concept de chaîne opératoire sont encore trop peu répandues dans les études lithiques en Asie du Sud-est continentale (Forestier 2000; Forestier et al. 2015; Jérémie \& Vacher 1992; White \& Gorman 2004; Zeitoun et al. 2008), contrairement aux approches morpho-typo ou statistiques (Marwick 2007, 2008b, 2013). En l'état, une utilisation raisonnable des méthodes statistiques devrait permettre d'éviter la radicalisation classificatoire des galets et sa conséquence totalisante qui en efface la variabilité et l'identité. 
Alors qu'au cours du Pléistocène supérieur final, l’Europe de l’Ouest, le Proche et le Moyen Orient connaissent l'apparition de nouveaux systèmes techniques lithiques et osseux avec une tendance à la généralisation d'outils spécialisés (grattoir, perçoir, burin, pointe, etc.) qui justifient le terme "d'émergence du Paléolithique supérieur et d'homme moderne », il n'en est rien en Asie du Sud-est. L'Asie tropicale ne connaîtra d'ailleurs jamais le débitage Levallois, ni le débitage laminaire pour la période des chasseurs-cueilleurs du Paléolithique final alors, qu'on entrevoit la production de lame seulement à une période récente en contexte d'ateliers de taille au Néolithique dans le Nord de la Thaïlande (Forestier et al. 2006).

Au sein du paradigme mondial de l'évolution des techniques lithiques, l'Asie du Sud-est fait ainsi figure d'exception et d'hapax technologique. Sur près de trente millénaires les hommes y ont élaboré un outillage de pierre (monotone) avec une panoplie d'outils peu variés, lourds, d'aspect " archaïque » sur galet largement dominés par une taille unifaciale. Il est difficile d'y déceler une tendance évolutive de l'outillage lithique comme c'est le cas notamment en Europe occidentale au Paléolithique supérieur, marqué par une grande diversité de supports-outils. Il convient aussi de noter que le Néolithique sud-est asiatique passe également pour être particulier. Assez tardif ce dernier est compris entre 4500 et 3500 BP et relèverait d'un phénomène côtier à partir de 6 000-5 000 BP, sous la forme de grands amas coquilliers dans les zones estuariennes comme par exemple, le site de Da But (Thanh Hoa) situé au sud du delta du Fleuve Rouge (Bellwood 2005: 131-134).

Quant au début de la culture du riz qui est présumée associée au Néolithique même si ce dernier n'est pas équivalent à l'avènement de l'agriculture partout dans le monde. Si cette culture est attestée très tôt vers 12000 ans BP en Chine dans des sites de la basse vallée du Yangtze (Wu et al. 2014), elle n'est connue que vers 3000 BP seulement en Thaïlande (Glover \& Higham 1996).

Faciès lithique du «Paléolithique supérieur extrême-oriental » le Hoabinhien est façonné par un homme moderne supposé posséder des capacités cognitives supérieures à celles de ses prédécesseurs ; capacités qui devraient alors être reflétées par les technologies et les matériaux qu'il met en œuvre en se démarquant d'un fond paléolithique plus ancien. C'est sans doute la raison pour laquelle ces industries sur galets ont longtemps porté préjudice à un Sud-est asiatique considéré comme isolé et retardataire par les préhistoriens.

Le Hoabinhien devrait au contraire constituer la preuve matérielle qu'une autre évolution des techniques sur le temps long est possible en dehors des productions de lames et de pointes en pierre ou en os. De toute évidence pour chasser, l'homme a projeté l'allongement et l'allègement doublé du nécessaire appointement de l'outillage dans d'autres matériaux plus éphémères, plus abondants et tout aussi efficaces, tirés du domaine végétal : le bambou (flexible-solide), le rotin, le bois dur et autres fibres abondantes dans ces régions tropicales humides (Bar-Yosef et al. 2012; Forestier 2010; Gorman 1970; Pope 1989; Solheim 1972; Testart 1977; West \& Louys 2007; Xhauflair 2014).

D’une manière plus générale les matières ligneuses répondent à des énergies plastiques que le préhistorien commence à peine à explorer par l'avancée des études fonctionnelles en tenant compte de facteurs nouveaux et contingents comme la déformation des matériaux (flexion, torsion, réaction, pli, etc.). Ou bien, s'agit-il déjà d'une nouvelle forme de " conception musculaire de la matière " pour reprendre une formule deleuzienne (Deleuze 1988: 6) dont on ne connaît pas encore très bien ni le contour, ni la portée ; et qui est, peutêtre, dans le cas du végétal, plus participante en terme d'instrumentalisation-instrumentation que dans celui du minéral ?

Alors que beaucoup de chercheurs s'interrogent sur les formes et les causes des transformations des sociétés préhistoriques au cours du temps dont les mécanismes nous échappent encore en Europe de l'Ouest ou au Proche Orient, en zone tropicale règne une autre aporie : l'inertie technique, la non évolution, le conservatisme, la stabilité, la monotonie voire 
l'apparente "somnolence » des industries lithiques. Sur ce point, accepter «l'inertie comme principe général (mais non absolu) » (Leroi-Gourhan 1945: 424), reviendrait dans notre cas à clore la réflexion sur l'objet technique dans un monde de chasseurs-cueilleurs résumé au « tout galet », et de facto, éluder la question des armes de chasse !

Comment se fait-il que ce faciès sur galet se perpétue sans pointe, sans apparent changement sur plusieurs dizaines de millénaires, sans marquer d'amélioration, d'inflexion ou de progrès et sans pour autant donner des signes de régression technique ? Serait-il alors un défi à la notion même d'évolution ainsi qu'à celle de progrès : un cas unique de stabilité et d’imperméabilité du milieu technique ?

La stabilité (ou, dans ce cas, l'inertie) du technocomplexe hoabinhien se présente avant tout comme le résultat logique d'une nécessité, d'une connivence entre les hommes et les lieux. Comme un dialogue étroit entre milieu intérieur et milieu environnant tropical et forestier : une solution originale trouvée, éprouvée et transmise aux groupes de chasseurs semi-nomades des forêts du Sud-est asiatique dès la fin du Pléistocène supérieur. L'outil générique sur galet ou uniface aux tranchants composites peut alors être interprété comme l'élément d'un toolkit hyper adapté aux conditions ou modes de vie, à un environnement de forêt pluviale à l'instar d'un «multipurpose survival tool » (Figure 19). Le rôle multivalent des tranchants fait de ces outils, des outils hybrides tournés vers la sphère du végétal, inépuisable source d'invention et d'innovation technique.

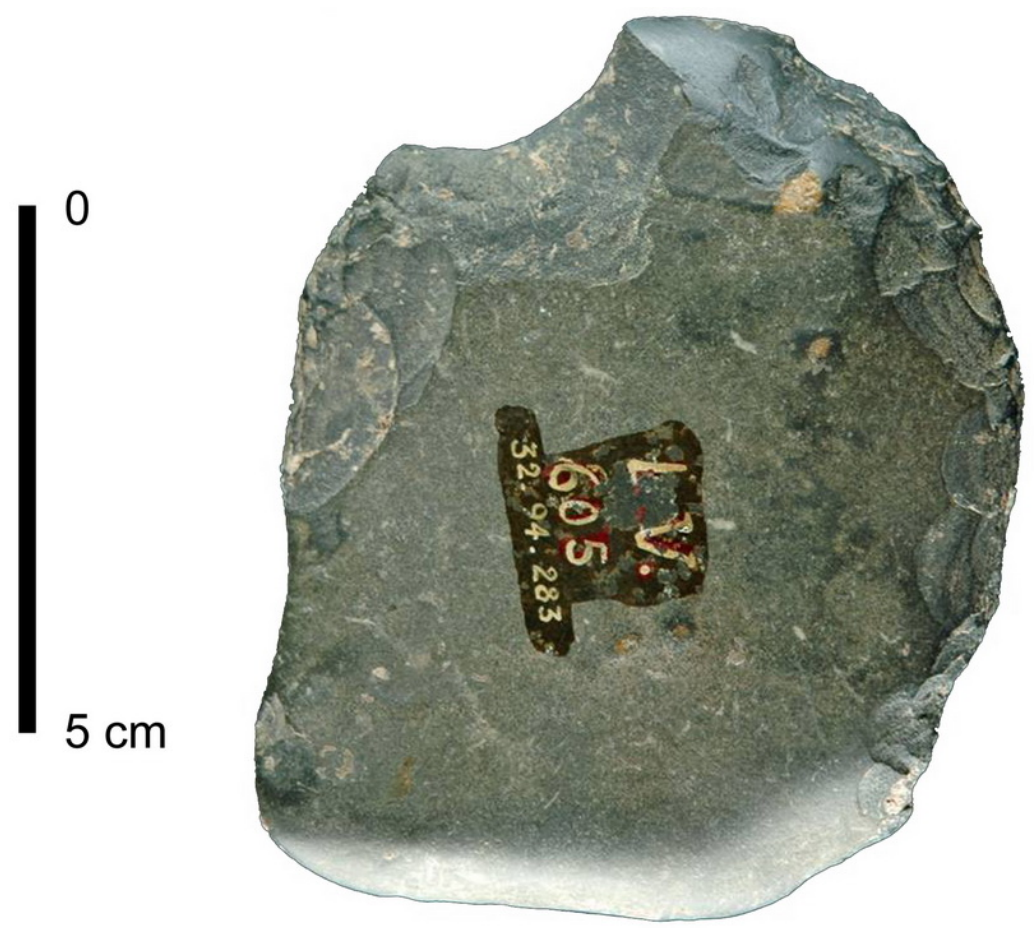

Figure 19. L'outil hoabinhien vu sous l'angle d'un outil de survie. (H. Forestier).

Figure 19. Hoabinhian tool view under the angle of a multipurpose survival tool concept. (H. Forestier).

\section{Vers l'ouverture d'un nouveau face-à-face : la pierre et son autre}

Le Hoabinhien emblématise et problématise une aire d'activités avec une dynamique autonome, interne mais aussi, probablement des réseaux encore mal connus qui demanderaient à être traduits sous la forme de variantes dans les modes de production (façonnage strict de galet sans débitage ; façonnage $\geq$ débitage ; façonnage $=$ débitage ; débitage $\geq$ façonnage, façonnage strict de plaquette, etc.). 
Dans ce monde technique sans rupture apparente, sans transition majeure au fil du temps et indissociablement lié à l'enracinement comme au déracinement par le voyage des gens du Hoabinhien jusqu'aux confins du monde péninsulaire indochinois (Sumatra et ses îles occidentales), existe-t-il des inflexions, des transformations ou une évolution interne par mutations discrètes de l'outillage (par exemple : apport et déterminisme de nouvelles matières premières comme le silex, la raréfaction des galets, utilisation partielle de la matière dure animale, etc.)?

Notre intérêt est à présent de chercher en direction des moteurs du changement d'une évolution interne au Hoabinhien (caractères discrets). Ce dernier se présente sous la forme d'un phénomène évolutif-additif sous-tendu par un renforcement progressif de la diversité, de la spécialisation et des savoir-faire impliqués dans la réalisation non pas de pointes de chasse qui restent très probablement en matière végétale périssable mais, d’une économie très précise de l'outillage et de la matière première lithique. Son émergence peut être vue comme une phase de globalisation culturelle à l'échelle de l'Asie du Sud-est, caractérisée par le développement autochtone d'une civilisation du végétal doublée d'une culture lithique tournée vers l'utilisation quasi-stricte du galet façonné dont, peut-être, la vocation première est d'être, dans ce cas, « au service du végétal ».

Ainsi, le milieu extérieur piloterait et expliquerait d'une certaine façon le degré d’homogénéité des productions lithiques. Le galet façonné et le petit outillage sur éclat associé cacheraient la complexité et la diversité d'outils (pointus et autres) en matière végétale, périssable et éphémère non conservés dans les sites archéologiques. Néanmoins quelques preuves existent comme des traces de phytolithes identifiées dans les niveaux du Pléistocène supérieur final de la grotte de Niah à Bornéo (Barker 2013) et dans un autre site de même époque sur l'île de Nouvelle-Bretagne en Papouasie (Lentfer et al. 2010). Des restes de fragments de bambou brulés auraient aussi été reconnus en présence d'os d'animaux non calcinés dans les niveaux hoabinhiens de la grotte Spirit cave en Thaïlande du Nord (Gorman 1969; 1970). A cela s'ajoute les nombreux résultats obtenus par l'avancée des études tracéologiques qui mettent en avant des évidences indirectes ou insinuées par la présence de micro-polis de végétaux (bois, bambou) au niveau des parties tranchantes des outils lithiques (Bannanurag 1988; Borel et al. 2013; Mijares 2008; Pawlik 2010; 2012; Pookajorn 1985; 2001; Reynolds et al. 2013; Xhauflair 2014; Xhauflair et al. 2016). Ces indices tirés d'observations archéologiques et analytiques plaident en faveur de l'existence logique d'un outillage végétal associé à la pierre dans ces régions tropicales. Si l'hypothèse tend à se confirmer pour l'homme moderne, elle est aussi envisagée pour les peuplements très anciens (Homo erectus) comme en témoigne une possible pièce en bambou fossilisée découverte dans un niveau de 800000 ans BP sur le site de Ngebung dans le dôme de Sangiran à Java central (Choi \& Driwantoro 2007; Sémah et al. 1992).

Tout cela porte à repenser plus largement la place de la pierre dans la technosphère des chasseurs-cueilleurs des forêts tropicales et de facto son rôle probablement secondaire dans les activités de chasse ou domestiques avec la découpe du gibier comme l'indique la documentation ethnographique (Hampton 1999; Pope 1989; Mijares 2001; Testart 1977). Dans cette perspective, les « couteaux » en bambou sont très largement utilisés dans de la plupart des sociétés traditionnelles des Philippines à la Papouasie (Revel 1990; Steenberg 1980; Xhauflair 2014); ainsi que les pointes, nombreuses en Irian-Jaya (Pétrequin \& Pétrequin 1990), en Malaisie (Dunn 1975), aux Philippines (Griffin 1997) ou en Indonésie occidentale (Forestier 2003; 2008; Forestier et al. 2008). A cela s’ajoutent les résultats de la microscopie électronique qui confirment la présence de traces particulières laissées par l'utilisation de ces couteaux sur des surfaces osseuses (Spennemann 1991; West 1989; West \& Louys 2007). Force est de constater que le travail des matières dures d'origine animale n'est que très peu répertorié dans les assemblages lithiques hoabinhiens alors qu'il est très 
présent en contexte insulaire où il est associé à un outillage sur petit éclat de débitage (Barton et al. 2013; Collings 1937; Forestier 1999; Forestier et al. 2010; Olsen \& Glover 2004; Marwick et al. 2016; Pasveer \& Bellwood 2004; Rabett 2005; 2012; Rabett et al. 2013; Van Heekeren 1972).

Deux mondes techniques lithiques se dessineraient en respectant la continentalité et l'insularité : un monde majoritairement sur galet dépourvu d'éléments pointus et à bords convergents (bord-pointe et bord-pointe-bord) remplacés par des matières végétales ; et un monde archipélagique centré sur de petites îles volcaniques (Indonésie et Philippines) à nombreux faciès sur éclat avec ou sans production de pointe. Le faciès Toalien de l'île indonésienne de Sulawesi s'inscrit précisément dans ces aires où se sont développées des industries lithiques à pointes de petite dimension (pointes dites de Maros) associées à des outils en os remontant à $7000 \mathrm{BP}$ (Duli et al. sous presse).

Insaisissable car disparu (pour l'heure) du champ de l'archéologie en Asie du Sud-est, l'identification de cet outillage en matière végétale transparaît dès lors que l'on replace le Hoabinhien dans la techno-sphère des chasseurs-cueilleurs de ces régions vivant à l'entrée des grottes, établissant des campements sous des abris sous-roches ou sur des collines, mais toujours dans un environnement de forêt tropicale humide.

Un cheminement du minéral au végétal qui fait écho à la nécessité de compléter « le toolkit » des artisans-chasseurs préhistoriques du Paléolithique final d'Asie du Sud-est par des objets pointus. Peut-on sérieusement envisager l'existence de groupes de chasseurs sans élément pointu (pierre ou autre) dans une région équivalente à la superficie de l'Europe occidentale?

Existe-t-il à l'échelle mondiale des groupes de chasseurs-cueilleurs-foragers sans pointe, sans projectile, sans objet angulaire, convergent ou acéré ? La réponse est non.

L'hypothèse d'une « civilisation du végétal » de P. Gourou (1948) ou celle d'un «âge du bambou » de G. Condominas (1978), maintes fois évoquée pour la préhistoire indochinoise trouverait confirmation dans l'étude des restes d'animaux aériens et terrestres chassés découverts à la fouille (archéozoologie), dans le domaine encore peu exploré de la tracéologie et enfin, dans un faisceau d'échanges noués autour du paradigme espace ou territoire dans lequel s'enracine le vécu des hommes. Les territoires du Hoabinhien demanderaient, sans doute, à être repensés dans le sens de l'aire d'extension du bambou (Figure 20) qui prolifère avec plus de 1000 espèces en Asie du Sud, de l'Est et du Sud-est en milieu de montagne (jusqu'à plus de 3000 m d'altitude dans l'Himalaya et ses contreforts) ou de plaine subtropicale à tropicale-équatoriale.

Au-delà des déterminismes géo-climatiques évidents du Sud-est asiatique, s'opèrent des choix culturels innovants et économiques émergents très largement reliés à la vie végétale, qui seraient un des nombreux aspects de la matérialisation de la «connivence des hommes et de leurs paysages » (Sautter 1979) : une autre définition de ce que l'on nomme Hoabinhien est alors possible dans laquelle le champ du lithique s’installerait à l'intérieur du milieu végétal.

Ces régions de forêts tropicales ont été les ateliers d'innovations techniques, d'objets insolites, originaux autant qu'inattendus, très loin de l'évolution des industries lithiques du Paléolithique final et Mésolithique d'Europe occidentale et ses périphéries.

En Asie du Sud-est, une autre évolution et une autre idée du progrès technique ont donc eu lieu, très éloignées d'une évolution linéaire des outillages du simple au complexe, du lourd au léger ; mais exprimant une préhistoire et une adaptation humaine qui, sur le temps long, ne ressemblent à aucune autre. C'est-à-dire au plus proche d'un contexte écologique tropical très particulier dans lequel, seul survivant, l'outil de pierre hoabinhien est aujourd'hui en mesure de réécrire la longue histoire de l'homme et du végétal, celle d'un galet qui cache la forêt et qui, contrairement au Néolithique, raconte une herbe plutôt qu'un arbre... ! 


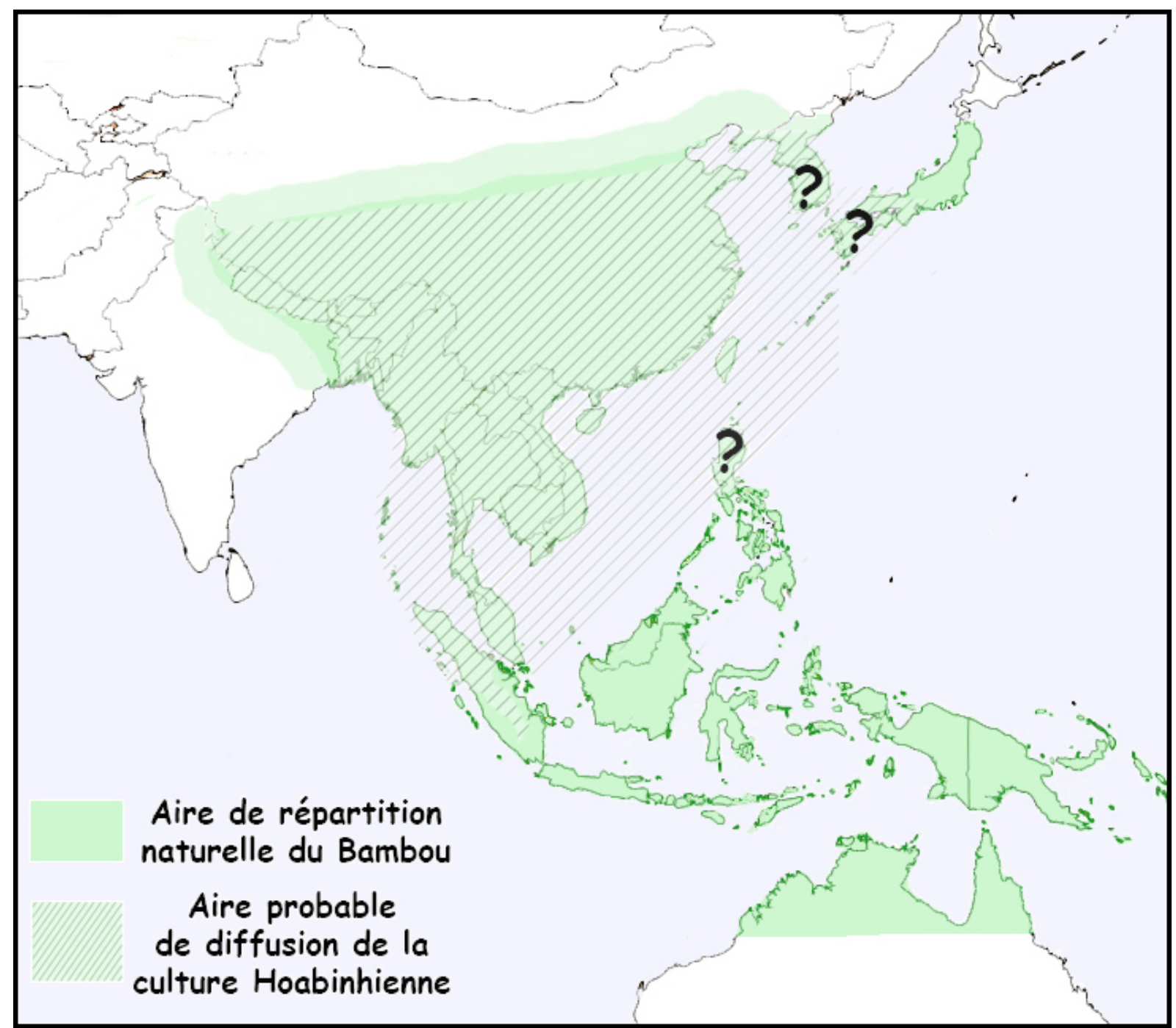

Figure 20. Aire de répartition du bambou et du Hoabinhien en Asie de l'Est et du Sud-Est.

Figure 20. Area of the bamboo and Hoabinhian distribution in East and Southeast Asia.

\section{Références}

Auetrakulvit, P., Forestier, H., Khaokhiew, C., \& Zeitoun, V. 2012, New excavation at Moh Khiew site, Southern Thailand. In: Crossing Borders: Selected papers from the 13th International Conference of the European Association of Southeast Asian

Archaeologists, Vol. 1 (Tjoa-Bonatz, M., Reinecke, A., \& Bonatz, D., Eds.), National University Singapore Press, Singapore: p. 60-71. (en anglais) ("Nouvelle fouille du site de Moh Khiew, sud de la Thaïlande”)

Bacon, A.M. (Ed.) 2012, Les sites de Tham Hang, Nam Lot et Tam Pà Ling au nord du Laos. Des gisements à vertébrés du Pléistocène aux origines des Hommes modernes. CNRS éditions, Paris, 149 p. (in French) ("The sites of Tham Hang, Nam Lot and Tam Pà Ling in northern Laos. From vertebrate sites from the Pleistocene to the origins of modern humans") 
Bannanurag, R. 1988, Evidence for ancient woodworking: A microwear study of Hoabinhian stone tools. In: Prehistoric studies: The stone and metal ages in Thailand (Charoenwongsa, P., \& Bronson, B., Eds.), The Thai Antiquity Working Group, Bangkok: p. 61-79. (en anglais) ("Preuves pour le travail du bois ancien : une étude tracéologique des outils en pierre du Hoabinhien”)

Barker, G. 2013, Rainforest foraging and farming in Island Southeast Asia. The archaeology of Niah Caves, Sarawak volume 1, McDonald Institute Monographs, McDonald Institute for Archaeological Research, Cambridge, 464 p. (en anglais) ("Forêt tropicale et agriculture dans l'île Asie du Sud-Est. L'archéologie de Niah Caves, Sarawak volume 1 ”)

Barton, H., Barker, G., Gilbertson, D., Hunt. C., Kealhofer, L., Lewis, H., Paz, V., Piper, J.P., Rabett, R.J., Reynolds, T., \& Szabo, K. 2013, Late Pleistocene foragers, c. 35 000-11 500 years ago (Chp.5). In: Rainforest foraging and farming in Island Southeast Asia. The archaeology of Niah Caves, Sarawak, Vol. 1 (Barker, G., Ed.), McDonald Institute Monographs, McDonald Institute for Archaeological Research, Cambridge: p. 173-215. (en anglais) ("Les cueilleurs du Pléistocène supérieur, il y a c. 35 000-11 500 ans”)

Bar-Yosef, O., Eren, M.I., Yuan, J., Cohen, D.J., \& Li, Y. 2012, Were bamboo tools made in prehistoric Southeast Asia? An experimental view from South China. Quaternary International, 269: 9-21. (en anglais) ("Des outils en bambou était-ils fabriqués en Asie du Sud-Est préhistorique? Une vue expérimentale de la Chine du Sud”) doi:10.1016/j.quaint.2011.03.026

Bellwood, P. 2005, The spread of agriculture into Southeast Asia and Oceania (Chap.7). In: First Farmers, The origins of agricultural societies (Bellwood, P., Ed.), Blackwell Publishing, Malden: p. 128-145. (en anglais) ("La propagation de l'agriculture en Asie du Sud-Est et en Océanie”)

Boëda, E. 2001, Détermination des unités techno-fonctionnelles de pièces bifaciales provenant de la couche acheuléenne C'3 base du site de Barbas I. In: Les industries à outils bifaciaux du Paléolithique moyen d'Europe Occidentale. Actes de la Table Ronde Internationale, Caen, 14-15 Octobre 1999 (Cliquet, D., Ed.), ERAUL 98, Université de Liège, Liège: p. 51-75. (in French) ("Determination of the techno-functional units of bifacial tools from the Acheulean layer C'3 base of the Barbas I site”)

Boëda, E. 2013, Techno-logique et Technologie. Une paléo-histoire des objets lithiques tranchants. Préhistoire au Présent, Editions @rchéoéditions.com, Paris, 259 p. (in French) ("Techno-logic and Technology. A palaeo-history of sharp lithic objects")

Bordes, F. 1950, L'évolution buissonnante des industries en Europe occidentale. Considération théoriques sur le Paléolithique ancien et moyen. L'Anthropologie, 54: 393-420. (in French) ("The Shifting Evolution of Industries from Western Europe. Theoretical Considerations on the Early and Middle Palaeolithic”)

Bordes, F. 1961, Typologie du Paléolithique ancien et moyen. Delmas, Bordeaux, 85 p. (in French) ("Early and Middle Palaeolithic Typology")

Bordes, F. 1974, A propos d'un biface sur dreikanter et d'un point de typologie. Bulletin de la Société Préhistorique Française, 71(6): 168-169. (in French) (“About a biface on dreikanter and a typology point”) doi:10.3406/bspf.1974.8318 
Borel, A., Gaillard, C., Moncel, M.H., Sala, R., Pouydebat, E., Simanjuntak, T., \& Sémah, F. 2013, How to interpret informal flakes assemblages? Integrating morphological description, usewear and morphometric analysis gave better understanding of the behaviors of anatomically modern human from Sog Terus (Indonesia). Journal of Anthropological Archaeology, 32: 630-646. (en anglais) (“Comment interpréter les assemblages de’éclats informels? L'intégration de la description morphologique, de la tracéologie et de l'analyse morphométrique a permis de mieux comprendre les comportements des humains anatomiquement modernes de Sog Terus (Indonésie)”) doi:10.1016/j.jaa.2013.03.002

Bowdler, S. 1994, The Hoabinhian in Australia: A retrospective review. Vietnam Social Sciences, 5(43): 87-94. (en anglais) ("Le Hoabinhien en Australie : une revue rétrospective")

Bowdler, S. 2006, The Hoabinhian: Early Evidence for Southeast Asian Trade Networks? In: Uncovering Southeast Asia's Past: Selected papers from the $10^{\text {th }}$ Annual Conference of the European Association of Southeast Asian Archaeologists (Bacus, E.A., Glover, I.C., \& Piggott, V., Eds.), National University of Singapore Press, Singapore: p. 355-359. (en anglais) ("The Hoabinhien : premières preuves pour les réseaux d'échanges de l'Asie du Sud-Est?”)

Brandt, R.W. 1976, The Hoabinhian of Sumatra: Some remarks. Modern Quaternary Research in Southeast Asia, 2: 49-52. (en anglais) ("Le Hoabinhien de Sumatra: quelques remarques”)

Bronson, B., \& Glover, I.C. 1984, Archaeological radiocarbon dates from Indonesia. Indonesia Circle, 34: 37-44. (en anglais) ("Datation au radiocarbone archéologiques d'Indonésie”)

Brown, P., Sutikna, T., Morwood, M.J., Soejono, R.P., Jatmiko, Saptomo, E.W., \& Due, R.A. 2004, A new small-bodied hominin from the Late Pleistocene of Flores, Indonesia. Nature, 43: 1055-1061. (en anglais) (“Un nouvel homininé à petite corpulence du Pléistocène supérieur de Flores, Indonésie”) doi:10.1038/nature02999

Bulmer, S. 1975, Settlement and economy in prehistoric Papua New Guinea: A review of the archaeological evidence. Journal de la Société des Océanistes, 46(31): 7-75. (en anglais) ("Implantations et économie en Papouasie-Nouvelle-Guinée préhistorique : un examen des preuves archéologiques”)

Cartailhac, E. 1877, L'âge de la pierre dans l'Indochine. Matériaux pour l'histoire primitive et naturelle de l'Homme, $2^{\mathrm{e}}$ série, 12(8): 98-100. (in French) ("The Stone Age in Indochina”) URL: http://gallica.bnf.fr/ark:/12148/bpt6k445325n

Choi, K., \& Driwantoro, D. 2007, Shell tool use by early members of Homo erectus in Sangiran central Java, Indonesia: Cut mark evidence. Journal of Archaeological Science, 34: 48-58. (en anglais) ("Utilisation d'outils en coquille par les premiers membres de Homo erectus à Sangiran Java central, Indonésie : preuve de marques de découpes”) doi:10.1016/j.jas.2006.03.013

Clark, G. 1969, World Prehistory: A New Synthesis. Cambridge University Press, Cambridge, 319 p. (en anglais) ("Préhistoire mondiale : une nouvelle synthèse”)

Colani, M. 1927, L’Âge de la Pierre dans la province de Hoa-Binh, Tonkin. Mémoires du service géologique d'Indochine (Hanoi), 14: 1-47. (in French) ("The Stone Age in HoaBinh Province, Tonkin”) 
Colani, M. 1929a, Quelques paléolithes hoabinhiens typiques de l'abri-sous-roche de Lang Kay. Bulletin de l'Ecole Française d'Extrême-Orient, 26: 353-384. (in French) ("Some typical Hoabinhian palaeoliths of the Lang Kay rock shelter”)

Colani, M. 1929b, Quelques stations hoabinhiennes (note préliminaire). Bulletin de l'Ecole Française d'Extrême-Orient, 29: 261-72. (in French) ("Some Hoabinhian Stations (Preliminary Note)")

Collectif. 1932, Praehistorica Asiae Orientalis : 1, Premier Congrès des préhistoriens d'Extrême-Orient, Hanoi. Imprimerie d'Extrême-Orient, Hanoi, 155 p. (in French) ("Praehistorica Asiae Orientalis: 1, First Congress of Prehistorians of the Far East, Hanoi”)

Collina-Girard, J. 1986, Grille descriptive et évolution typologique des industries archaïques : le modèle catalan. Bulletin de la Société Préhistorique Française, 83(11-12): 383-403. (in French) ("Descriptive grid and typological evolution of archaic industries: The Catalan model”) doi:10.3406/bspf.1986.8716

Collings, H.D. 1937, An excavation at Bukit Chuping, Perlis. Bulletin of the Raffles Museum, Series B, 1(2): 94-119. (en anglais) (“Une fouille à Bukit Chuping, Perlis”)

Condominas, G. 1978, L’Asie du Sud-Est. In: Ethnologie Régionale II (Poirier, J., Ed.). Gallimard (La Pléiade), Paris: p. 309-310. (in French) (“Southeast Asia”)

Deleuze, G. 1988, Le Pli. Leibniz et le baroque. Collection Critique, Les éditions de Minuit, Paris, 192 p. (in French) (“The Fold. Leibniz and Baroque ”)

Duli, A., Chia, S., Nur, M., Suryatman, Saiful, A.M., Hasanuddin., Hakim, B., Supriadi., Rosmawati., Somba, N., Bernadeta sous presse, A new perspective about maros point layer from Panningnge cave, Maros, Indonesia. Bulletin of Indo-Pacific Prehistory Association, 15 p. (en anglais) ("Une nouvelle perspective sur la couche de pointes maros de la grotte de Panningnge, Maros, Indonésie”)

Dunn, F.L. 1975, Rain-forest collectors and traders. A study of resource utilization in modern and ancient Malaya. MBRAS Press, Kuala Lumpur, Malaysia, 151 p. (en anglais) ("Collectionneurs et commerçants de la forêt tropicale ")

Faivre, J.P., Geneste, J.M., \& Turq, A. 2010, La fracturation en split, une technique de production dans l'industrie lithique des Tares (Sourzac, Dordogne). Paléo. Revue d'archéologie préhistorique [Online], Numéro special, 2009-2010, Online since 12 April 2012, Last viewed on 12 January 2016. (in French) ("Split fracture, a production technique in the lithic industry of Tares (Sourzac, Dordogne)”)

URL: http://paleo.revues.org/1944

Forestier, H. 1999, L'assemblage industriel de Song Keplek, Java Est : un nouveau regard sur l'outillage de l'homme moderne au début de l'Holocène en Indonésie. Bulletin de l'Ecole française d'Extrême-Orient, 86(1): 129-159. (in French) (“The Song Keplek Industrial Assembly, East Java: A New Look at the Tooling of Modern Man in the Early Holocene in Indonesia”) doi:10.3406/befeo.1999.3409

Forestier, H. 2000, De quelques chaînes opératoires lithiques en Asie du Sud-Est au Pléistocène supérieur final et au début de l'Holocène. L'Anthropologie, 104: 531-548. (in French) ("From some lithic chaînes opératoires in South-East Asia during the late Upper Pleistocene and early Holocene”) doi:10.1016/S0003-5521(00)80025-4 
Forestier, H. 2003, Des outils nés de la forêt. L’importance du végétal en Asie du Sud-Est dans l'imagination et l'invention technique aux périodes préhistoriques. In:

Peuplements anciens et actuels des forêts tropicales, Colloques et séminaires, Octobre 1998 (Froment, A., \& Guffroy, J., Eds.), Ermes-IRD, Paris: p. 315-337. (in French) ("Tools born of the forest. The importance of plant in Southeast Asia in the imagination and technical invention during prehistoric times”)

Forestier, H. 2008, Les dessous de l'espace. Un dialogue archéologique entre le temps, les techniques et l'espace. EcoGéo, 5: 1-8. (in French) ("The underside of space. An archaeological dialogue between time, techniques and space”) doi:10.4000/echogeo.4548

Forestier, H. 2010, La pierre et son ombre. Réflexion sur le phénomène hoabinhien d'Asie du Sud-Est. Habilitation à Diriger des Recherches, Université de Paris Ouest-Nanterre La Défense, Paris, 168 p. (in French) ("The stone and its shadow. Reflection on the Hoabinhian phenomenon of Southeast Asia”)

Forestier, H. 2014, New World, new models. Antiquity, 88(341): 945-948. (en anglais) (“Nouveau Monde, nouveaux modèles”) doi:10.1017/S0003598X00050870

Forestier, H., Simanjuntak, H.T., Guillaud, D., Driwantoro, D., Wiradnyana, K., Siregar, D., Due Awe, R., \& Budiman. 2005a, Le site de Tögi Ndrawa, île de Nias, Sumatra nord : les premières traces d'une occupation hoabinhienne en grotte en Indonésie. Comptes Rendus Palevol, 4: 727-733. (in French) ("The Tögi Ndrawa Site, Nias Island, North Sumatra: The first traces of Hoabinhian cave occupation in Indonesia”) doi:10.1016/j.crpv.2005.08.004

Forestier, H., Zeitoun, V., Seveau, A., Driwantoro, D., \& Winayalai, C. 2005b, Prospections paléolithiques et perspectives technologiques pour redéfinir le Hoabinhien du nord de la Thaïlande (campagnes 2002-2005). Aséanie, 15: 33-60. (in French) ("Palaeolithic Prospects and Technological Prospects for Redefining Hoabinhien in Northern Thailand (2002-2005 Campaigns)”) doi:10.3406/asean.2005.1845

Forestier, H., Zeitoun, V., Tiamtinkit, C., Boonyai, S., Winayalai, C., \& Auetrakulvit, P. 2006, Lames du Néolithique dans les carrières de Nan (Nord de la Thaïlande). Aséanie, 18: 46-89. (in French) ("Neolithic Blades in the Nan Quarries (Northern Thailand)”)

Forestier, H., Guillaud, D., Meyers, K., \& Simanjuntak, T., 2008, Mentawai : l’île des hommes fleurs. Romain Pages Éditions, Sommières, 152 p. (in French) (“Mentawai: The Isle of Men Flowers”)

Forestier, H., Simanjuntak, H.-T., Detroit, F., \& Zeitoun, V. 2010, Unité et diversité préhistorique entre Java et Sumatra. Archipel, 80: 19-44. (in French) ("Unity and prehistoric diversity between Java and Sumatra. Archipelago”)

Forestier, H., Sophady, H., Zeitoun, V., \& Mourer, R. 2012, Le Cambodge avant l'Histoire : aux origines de la préhistoire Khmère. In: Orientalismes. De l'archéologie au musée, Mélanges offerts à Jean-François Jarrige (Lefèvre, V., Ed.), Brepols Publishers, Turnhout: p. 193-212. (in French) (“Cambodia before history: The origins of Khmer prehistory") 
Forestier, H., Zeitoun, V., Winayalai, C., \& Métais, C. 2013, The open-air site of Huai Hin (Northwestern Thailand): Chronological perspectives for the Hoabinhian. Comptes Rendus Palevol, 12: 45-55. (en anglais) ("Le site en plein air de Huai Hin (Nord-Ouest de la Thaïlande) : Perspectives chronologiques pour le Hoabinhien”) doi:10.1016/j.crpv.2012.09.003

Forestier, H., Sophady, H., Puaud, S., Mourer, R., Billault, L., Philippe, M., \& Zeitoun, V. 2014, New evidence of old stone tools from the Mekong terraces, Cambodia. Comptes Rendus Palevol, 13: 109-120. (en anglais) ("Nouvelle preuve de vieux outils de pierre des terrasses du Mékong, Cambodge”) doi:10.1016/j.crpv.2013.09.006

Forestier, H., Sophady, H., Puaud, S., Celiberti, V., Frère, S., Zeitoun, V., Mourer-Chauviré C., Mourer, R., Than, H., \& Billault, L. 2015, The Hoabinhian from Laang Spean Cave in its stratigraphic, chronological, typo-technological and environmental context (Cambodia, Battambang province). Journal of Archaeological Science: Reports, 3: 194206. (en anglais) ("Le Hoabinhien de la grotte Laang Spean dans son contexte stratigraphique, chronologique, typo-technologique et environnemental (Cambodge, province de Battambang)”) doi:10.1016/j.jasrep.2015.06.008

Fromaget, J. 1940, La stratigraphie des dépôts préhistoriques de Tam Hang (Chaîne Annamitique septentrionale) et ses difficultés. In: Proceedings of the Third Congress of Prehistorians of the Far East, Singapore 1938 (Chasen, F., \& Tweedie, M.W.F., Eds), Congress of Prehistorians of the Far East, Singapour: p. 60-70. (in French) ("The stratigraphy of the prehistoric deposits of Tam Hang (Northern Annamite Chain) and its difficulties”)

Glover, I.C., \& Higham, C.F. 1996, New evidence for Early rice cultivation. In: The origins and spread of agriculture and pastoralism in Eurasia (Harris, D., Ed.), UCL Press, London: p. 412-44. (en anglais) ("Nouvelle évidence pour la culture du riz précoce”)

Gorman, C. 1969, Hoabinhian: A pebble tool complex with early plant associations in Southeast Asia. Science, 163: 671-673. (en anglais) ("Hoabinhien : Un complexe de galets taillés avec des associations végétales anciennes en Asie du Sud-Est”) URL: http://www.jstor.org/stable/1726336

Gorman, C. 1970, Excavation at Spirit cave, North Thailand. Asian Perspectives, 13: 79-107. (en anglais) ("Fouilles à la grotte de Spirit, nord de la Thaïlande ”)

Gourou, P. 1948, La civilisation du végétal. Indonésie, 1(5): 385-396. (in French) (“The civilization of the plant")

Groube, L., Chapell, J., Muke, J., \& Price, D. 1986, A 40000 years-old human occupation site at Huon peninsula, Papua New Guinea. Nature, 324: 453-455. (en anglais) ("Un site d'occupation humaine de 40000 ans dans la péninsule de Huon, Papouasie-NouvelleGuinée”) doi:10.1038/324453a0

Griffin, P.B. 1997. Technology and variation in arrow design among the Agta of Northeastern Luzon, In: Projectile Technology (Knecht, H., Ed.), Springer, New York: p. 267-286. (en anglais) ("Technologie et variation dans la conception des flèches dans l'Agta du Nord-Est de Luçon”)

Hampton, O.W.B. 1999, Culture of Stone: Sacred and profane uses of stone among the Dani. Texas A\&M University Anthropology Series, Texas A\&M University Press, College Station, 360 p. (in French) (“Culture of Stone: Sacred and profane uses of stone among the Dani”) 
Harrisson, T. 1957, The great cave of Niah: Preliminary report. Man, 59: 161-166. (en anglais) ("La grande caverne de Niah : rapport préliminaire”)

Harrisson, T. 1963, 100000 years of Stone Age culture in Borneo. Journal of the Royal Society of Art, 112: 74-91. (en Anglais) (“100 000 ans de culture de l'âge de pierre à Bornéo")

Harrisson, T. 1970, The Prehistory of Borneo. Asian Perspectives, 13: 17-46. (en anglais) ("La préhistoire de Bornéo ”)

Hazarika, M. 2011, Prehistoric cultural affinities between Southeast Asia, East Asia and Northeast India: An exploration. In: Unearthing Southeast Asia's Past: Selected papers from the $12^{\text {th }}$ International Conference of the European Association of Southeast Asian Archaeologists (Klokke, M.J., \& Degroot, V., Eds.), National University Singapore Press, Singapore: p. 16-25. (en anglais) ("Les affinités culturelles préhistoriques entre l'Asie du Sud-Est, l'Asie de l'Est et l'Inde du Nord-Est : une exploration”)

Heekeren, H.R. Van. 1972, The stone age of Indonesia ( $2^{\text {nd }}$ edition). Martinus Nijhoff, The Hague, 311 p. (en anglais) ("L'âge de pierre de l'Indonésie”)

Higham, C. 2013, Hunter-Gatherers in Southeast Asia: From Prehistory to the Present. Human Biology, 85(1-3): 21-44. (en anglais) (“Chasseurs-cueilleurs en Asie du SudEst : De la Préhistoire à nos jours”)

Inizan, M.L. 1976, Nouvelle étude d'industries lithiques du Capsien. Thèse de $3^{\text {ème }}$ cycle, Université de Paris X - Nanterre, Nanterre, 328 p. (in French) ("New study of Capsian lithic industries”)

Jérémie, S., \& Vacher, S. 1992, Le Hoabinhien en Thaïlande : un exemple d'approche expérimentale. Bulletin de l'Ecole Française d'Extrême-Orient, 79(1): 173-209. (in French) ("The Hoabinhian in Thailand: An Example of an Experimental Approach")

Khairuddin, A. 2010, Adaptasi pesisiran pantai masyarakat Hoabihnian di Malaysia Barat: Satu catatan ringkas. Jurnal Arkeologi Malaysia, 24: 1-8. (en indonésien) (“Adaptation côtière des populations hoabiniennes dans l'ouest de la Malaisie : une vue courte ”)

Lentfer, C., Pavlides, C., \& Specht, J. 2010, Natural and human impact in a 35000 year vegetation history in Central New Britain, Papua New Guinea. Quaternary Science Review, 29: 3750-3767. (en anglais) ("Impact naturel et humain dans une histoire de végétation de 35000 ans dans le centre de la Nouvelle-Bretagne, Papouasie-NouvelleGuinée”) doi:10.1016/j.quascirev.2010.08.009

Le Brun-Ricalens, F. 1989, Un type particulier de galet aménagé dans certaines industries à quartzites taillés de la moyenne Garonne : «l'uniface à dos ». Bulletin de la Société Méridionale de Spéléologie et de Préhistoire, 29: 17-21. (in French) (“A particular type of cobble-stone worked in some quartzite industries from the Middle Garonne: "The backed uniface"'”)

Leroi-Gourhan, A. 1945, Evolution et techniques. II. Milieu et techniques. Albin Michel, Paris, 475 p. (in French) (“Evolution and techniques. II. Environment and techniques”)

Lepot, M. 1993, Approche techno fonctionnelle de l'outillage lithique moustérien : essai de classification des parties actives en termes d'efficacité technique. Mémoire de Maîtrise de l’Université de Paris X-Nanterre, Nanterre, 249 p. (in French) ("Technical approach to Mousterian lithic tools: Test of classification of the active parts in terms of technical efficiency") 
Majid, Z. 2003, Arkeologi di Malaysia. Centre for Archaeological Research, Sains University Malaysia, 208 p. (en malais) (“Archéologie de la Malaisie”)

Maringer, J. 1957, Some stone toll of Early Hoabinhian type from Central Japan. Man, 57: 14. (en anglais) (“Un peu de pierre de type Hoabinhien Ancien du Japon central”)

Marwick, B. 2007, Approaches to flaked stone artefact Archaeology in Thailand: A Historical Review. Silpakorn University International Journal, 7: 49-88. (en anglais) (“Approches sur l'outillage sur éclat de Thaïlande : un récapitulatif historique”)

Marwick, B. 2008a, Stone artifact and human ecology at two Rockshelters in Northwest Thailand. Unpublished Ph.D., Archaeology and Natural History, The Australian National University, Canberra, 295 p. (en anglais) (“Outillage en pierre et écologie humaine dans deux abris sous roche dans le nord-ouest de la Thaïlande”)

Marwick, B. 2008b, What attributes are important for the measurement of assemblage reduction intensity? Results from an experimental stone artefact assemblage with relevance to the Hoabinhian of mainland Southeast Asia. Journal of Archaeological Science, 35: 1189-1200. (en anglais) ("Quels attributs sont importants pour la mesure de l'intensité de réduction de l'assemblage? Résultats d'un assemblage expérimental d'artefacts en pierre pertinent au Hoabinhien de l'Asie du Sud-Est continentale”) doi:10.1016/j.jas.2007.08.007

Marwick, B. 2013, Multiple Optima in Hoabinhian flaked stone artefact palaeoeconomics and palaeoecology at two archaeological sites in Northwest Thailand. Journal of Anthropological Archaeology, 32: 553-564. (en anglais) (“Optima Multiple dans la paléoéconomie et paléoécologie de l’outillage en pierre taillé du Hoabinhien dans deux sites de Thaïlande du Nord-Ouest”) doi:10.1016/j.jaa.2013.08.004

Marwick, B., Clarkson, C., O’Connor, S., \& Collins, S. 2016, Early modern human lithic technology from Jerimalai, East Timor. Journal of Human Evolution, 101: 45-64. (en anglais) ("La technologie lithique chez les humains modernes de Jerimalai, Timor oriental”) doi:10.1016/j.jhevol.2016.09.004

Matthews, J.M. 1960, A note on the rock paintings recently discovered near Ipoh, Perak. Man, 60: 1-3. (en anglais) ("Une note sur les peintures rupestres récemment découvert près d'Ipoh, Perak")

Matthews, J.M. 1966, The Hoabinhian affinities of some Australian assemblages. Archaeology and Physical Anthropology in Oceania, 1: 5-22. (en anglais) ("Les affinités hoabinhiennes de certains assemblages australiens”)

Maxwell, A.L. 2001, Holocene monsoon changes inferred from lake sediment pollen and carbonate records, Northeastern Cambodia. Quaternary Research, 56(3): 390-400. (en anglais) ("Changements de mousson de l'Holocène déduits des enregistrements de pollen et de carbonate de sédiments lacustres, dans le nord-est du Cambodge”) doi:10.1006/qres.2001.2271

McKinnon, E.E. 1990, The Hoabinhian in the Wampu/Lau Biang valley of Northeastern Sumatra: An update. Bulletin of the Indo-Pacific Prehistory Association, 10: 132-142. (en anglais) ("The Hoabinhien dans la vallée Wampu / Lau Biang du nord-est de Sumatra : une mise à jour”)

Mijares, A.S. 2001, An expedient lithic technology in Northern Luzon (Philippines). Lithic Technology, 26: 138-152. (en anglais) ("Une technologie lithique expéditive dans le nord de Luzon (Philippines)”) doi:10.1080/01977261.2001.11720983 
Mijares, A.S. 2008, The Late Pleistocene to Early Holocene foragers of Northern Luzon. Bulletin of the Indo-Pacific Prehistory Association, 28: 99-107. (en anglais) ("Les cueilleurs du Pléistocène supérieur au début de l'Holocène dans le nord de Luçon”)

Mohanty, P., Basa, K., \& Tripathy, B. 1997, Stone Age cultures of Mayurbhanj district, Orissa. Man in India, 77: 159-177. (en anglais) ("Les cultures de l'âge de pierre du district de Mayurbhanj, Orissa”)

Moser, J. 2001, Hoabinhian: Geographie und Chronologie eines steinzeitlichen Technokomplexes in Südostasien. Linden Soft, Cologne, 194 p. (en allemand) (“Hoabinhien : Géographie et chronologie d'un technocomplexe de l’Âge de la pierre en Asie du Sudest”)

Mourer, C., \& Mourer, R. 1970, The prehistoric industry of Laang Spean, province of Battambang, Cambodia. Archaeology and Physical Anthropology in Oceania, 5: 128146. (en anglais) ("L'industrie préhistorique de Laang Spean, province de Battambang, Cambodge”)

Mourer, C., \& Mourer, R. 1971, Prehistoric research in Cambodia during the last ten years. Asian Perspectives, 14: 35-42. (en anglais) ("La recherche préhistorique au Cambodge au cours des dix dernières années”)

Mourer, R. 1994, Contribution à l'étude de la préhistoire du Cambodge. In: Etudes thématiques I. Recherches nouvelles sur le Cambodge (Bizot, F., Ed.), Ecole Française d'Extrême Orient, Paris: p.143-195. (in French) ("Contribution to the study of prehistory of Cambodia”)

Movius, H.L. 1948, The Lower Palaeolithic cultures of Southern and Eastern Asia. Transations of the American Philosophical Society, 38(4): 329-420. (en anglais) ("Les cultures du Paléolithique inférieur de l'Asie du Sud et de l'Est”)

Olsen, S.L., \& Glover, I.C. 2004, The bone industry of Ulu Lean 1 and Leang Burung 1 rockshelters, Sulawesi, Indonesia, in its regional context. Modern Quaternary Research in Southeast Asia, 18: 273-299. (en anglais) ("L'industrie osseuse des abris sous roche de Ulu Lean 1 et Leang Burung 1, Sulawesi, en Indonésie, dans son contexte régional”)

Pasveer, J.M., \& Bellwood, P. 2004, Prehistoric bone artefacts from northern Moluccas, Indonesia. Modern Quaternary Research in Southeast Asia, 18: 301-359. (en anglais) (“Objets osseux préhistoriques du nord des Moluques, Indonésie”)

Pawlik, A.F. 2010, Have we overlooked something? Hafting traces and indications of modern traits in the Philippine Palaeolithic. Bulletin of the Indo-Pacific Prehistory Association, 30: 35-53. (en anglais) (“Avons-nous oublié quelque chose? Traces d'emmanchement et des indications de traits modernes dans le Paléolithique des Philippines”)

Pawlik, A.F. 2012, Behavioural complexity and modern traits in the Philippine Upper Palaeolithic. Asian Perspectives, 51: 22-46. (en anglais) (“Complexité comportementale et traits modernes du Paléolithique supérieur philippin”)

Pearson, R. 1976, The contribution of archaeology to Japanese studies. Journal of Japanese Studies, 2(2): 305-333. (en anglais) (“La contribution de l'archéologie aux études japonaises”) 
Penny, D. 2001, A 40,000 years palynological record from north-east Thailand; implications for biogeography and palaeo-environmental reconstruction. Palaeogeography, Palaeoclimatology, Palaeoecology, 171(3-4): 97-128. (en anglais) (“Un spectre palynologique de 40.000 ans du nord-est de la Thaïlande; implications pour la biogéographie et la reconstruction paléo-environnementale”) doi:10.1016/S00310182(01)00242-5

Penny, D. 2006, The Holocene history and development of the Tonle Sap, Cambodia. Quaternary Science Reviews, 25: 310-322. (en anglais) ("L'histoire de l'Holocène et le développement du Tonlé Sap, Cambodge”) doi:10.1016/j.quascirev.2005.03.012

Perlès, C. 1980, Economie de la matière première et économie de débitage : deux exemples grecs. Préhistoire et technologie lithique. Cahier de l'URA (Unité de Recherche Associée), 28(1): 37-41. (in French) ("Economics of the raw material and economy of debitage: Two Greek examples”)

Pétrequin, P., \& Pétrequin, A.M. 1990, Flèches de chasse, flèches de guerre, le cas des Dani d’Irian Jaya (Indonésie). Bulletin de la Société préhistorique française, 87: 484-511. (in French) ("Hunting arrows, war arrows, the case of the Dani of Irian Jaya (Indonesia)") doi:10.3406/bspf.1990.9931

Pigeot, N. 1991, Réflexions sur l'histoire technique de l'homme : de l'évolution cognitive à l'évolution culturelle. Paléo, 3: 167-200. (in French) ("Reflections on the technical history of man: From cognitive evolution to cultural evolution") doi:10.3406/pal.1991.1046

Pookajorn, S. 1985, The technological and functional morphology analysis of the lithic tools from the hoabinhian excavation at Ban Kao area, Kanchenaburi Province, Thailand. Faculty of Archaeology, Silpakorn University Press, Bangkok, 185 p. (en anglais) ("L'analyse de la morphologie technologique et fonctionnelle des outils lithiques de la fouille hoabinhienne dans la région de Ban Kao, province de Kanchenaburi, Thaïlande”)

Pookajorn, S. 1988, Archaeological research of the Hoabinhian culture technocomplex and its comparison with ethnoarchaeology of the Phi Tong Luang, a hunter-gatherer group of Thailand. Archaeologia Verlag Band 9, Institut für Urgeschichte der Universität Tübingen, Tübingen, 349 p. (en anglais) ("Recherche archéologique du technocomplex de la culture Hoabinhien et sa comparaison avec l'ethnoarchéologie du Phi Tong Luang, un groupe de chasseurs-cueilleurs de Thaïlande”)

Pookajorn, S. 2001, New perspectives for Palaeolithic research in Thailand. In: Origine des peuplements et chronologie des cultures paléolithiques du Sud-est asiatique, Colloque International de la Fondation Singer-Polignac, Paris du 3 au 5 juin 1998 (Sémah, F., Falguères, C., Grimaud-Hervé, D., \& Sémah, A.M., Eds.), Editions Artcom’, Paris: p. 167-187. (en anglais) ("Nouvelles perspectives pour la recherche paléolithique en Thaïlande")

Pope, G.G., Nakbunlung, S., \& Pitragool, S. 1987, Le Paléolithique du Nord de la Thaïlande. Découvertes et perspectives nouvelles. L'Anthropologie, 91(3): 749-754. (in French) ("The Palaeolithic of Northern Thailand. Discoveries and new perspectives")

Pope, G.G. 1989, Bamboo and human evolution. Natural History, 10: 49-57. (en anglais) (“L'évolution du bambou et de l'homme”) 
Rabett, R.J. 2005, The early exploitation of Southeast Asian mangroves: Bone technology from caves and open sites. Asian Perspectives, 44(1): 154-179. (en anglais) ("L'exploitation ancienne des mangroves de l'Asie du Sud-Est : la technologie osseuse d'après les grottes et sites de plein air”)

Rabett, R.J. 2012, Human adaptation in the Asian Palaeolithic. Hominid dispersal and behaviour during the Late Quaternary. Cambridge University Press, Cambridge, 372 p. (en anglais) ("L'adaptation humaine au paléolithique asiatique. Dissémination des hominidés et comportement au cours du Quaternaire final”)

Rabett, R.J., Barker, G., Hung, G.O., Naruse, T., Piper, P., Raddatz, E., Reynolds, T., Nguyen Van Son., Strimpson, C., Szabo, K., Nguyen Cao Tan., \& Wilson, J. 2009, The Trang An project: Late to post Pleistocene settlement of the Lower Song Hong Valley, North Vietnam. Journal of the Royal Asiatic Society, 19: 73-109. (en anglais) ("Le projet Trang An : Colonisation de la fin et post Pléistocène dans la vallée de Lower Song Hong, au nord du Vietnam”)

Rabett, R.J., Barker, G., Barton, H., Hunt. C., Lloyd-Smith, L., Paz, V., Piper, P., Premathilake, R., Rushworth, G., Stephens, M., \& Szabo, K. 2013, Landscape transformations and human responses c.11 500 - c.4 500 years ago (Chp.6). In: Rainforest foraging and farming in Island Southeast Asia. The archaeology of Niah Caves, Sarawak, Vol. 1 (Barker, G., Ed.), McDonald Institute Monographs, McDonald Institute for Archaeological Research, Cambridge: p. 217-253. (en anglais) (“Transformations des paysage et réponses humaines de c.11 500 à c.4 500 ans”)

Rahman, M. 2002, The Hoabinhian site at Taat Hill Cave, upper Terengganu, Malaysia. Jebat, 29: 69-78. (en anglais) ("Le site Hoabinhien à Taat Hill Cave, Upper Terengganu, Malaisie”)

Revel, N. 1990, Fleurs de paroles. Histoire naturelle de Palawan II. La maitrise d'un savoir faire et l'art d'une relation. Editions Peeters, Paris, 350 p. (in French) ("Flowers of Words. Natural History of Palawan II. The mastery of a know-how and the art of a relationship")

Reynolds, T.E.G. 1990, Problems in the Stone Age of Thailand. Journal of the Siam Society, 78: 109-114. (en anglais) ("Problèmes à l'âge de pierre en Thaïlande”)

Reynolds, T.E.G., Barker, G., Barton, H., Granbrook, G., Hunt, C., Kealhofer, L;, Pike, A.W.G., Piper, P., Rabett, R., Rushworth, G., Stimpson, C., \& Szabo, K. 2013. The first modern humans at Niah, c. 50,000-35,000 years ago. In: Rainforest foraging and farming in Island Southeast Asia. The archaeology of Niah Caves, Sarawak, Vol. 1 (Barker, G., Ed.), McDonald Institute Monographs, McDonald Institute for Archaeological Research, Cambridge: p. 135-172. (en anglais) ("Les premiers humains modernes à Niah, entre c. 50000 et 35000 ans”)

Saurin, E. 1966, Le mobilier préhistorique de l'abri-sous-roche de Tam Pong (Haut Laos). Bulletin de la Société des Etudes d'Indochine, 41: 106-18. (in French) (“The prehistoric artefacts from the Tam Pong rock shelter (Upper Laos)")

Sautter, G. 1979. Le paysage comme connivance. Hérodote, 16: 40-67. (in French) (“The landscape as connivance”) 
Schoocongdej, R. 2006, Late Pleistocene activities at the Tham Lod Rockshelter in Highland Pang Mapha, Mae Hong Son Province, Northwestern Thailand. In: Uncovering Southeast Asia's Past: Selected papers from the $10^{\text {th }}$ Annual Conference of the European Association of Southeast Asian Archaeologists (Bacus, E.A., Glover, I.C., \& Piggott, V., Eds.), National University Singapore Press, Singapore: p. 22-37. (en anglais) (“Activités du Pléistocène supérieur à l'abri sous roche de Tham Lod Rockshelter à Highland Pang Mapha, Province de Mae Hong Son, Nord-Ouest de la Thaïlande")

Sémah, F. 2001, La position stratigraphique du site de Ngebung2 (dôme de Sangiran, Java Central, Indonésie). In: Origine des peuplements et chronologie des cultures paléolithiques du Sud-est asiatique, Colloque International de la Fondation SingerPolignac, Paris du 3 au 5 juin 1998 (Sémah, F., Falguères, C., Grimaud-Hervé, D., \& Sémah, A.M., Eds.), Editions Artcom’, Paris: p. 299-329. (in French) (“The stratigraphic position of the Ngebung2 site (Sangiran dome, Central Java, Indonesia)”)

Sémah, F., Sémah, A.M., Djubiantono, T., \& Simanjuntak, H.T. 1992, Did they also make stone tools? Journal of Human Evolution, 23: 439-446. (en anglais) (“Ont-ils également fabriqué des outils en pierre?”) doi:10.1016/0047-2484(92)90092-N

Sémah, F., Simanjuntak, H.T., Dizon, E., Gaillard, C., \& Sémah, A.M. 2014, Insular Southeast Asia in the Lower Palaeolithic. In: Encyclopedia of Global Archaeology (Smith, C., Ed.), Springer Publisher, New York: p. 3904-3918. (en anglais) (“Asie insulaire du sud-est au Paléolithique inférieur”)

Shea, J.J. 2013, Lithic Modes A-I: A New Framework for Describing Global-Scale Variation in Stone Tool Technology Illustrated with Evidence from the East Mediterranean Levant. Journal of Archaeological Method and Theory, 20(1):151-186. (en anglais) ("Modes lithiques A-I : Un nouveau cadre pour décrire la variation à l'échelle mondiale de la technologie des outils en pierre illustrée par des données probantes provenant du Levant méditerranéen oriental”) doi:10.1007/s10816-012-9128-5

Sieveking, G. 1954, Excavations at Gua Cha, Kelatan (part1). Federation Museums Journal, 1-2: 75-128. (en anglais) (“Fouilles à Gua Cha, Kelatan (partie 1)”)

Soejono, R.P. 1962, Indonesia. Asian Perspectives, 6: 34-43. (en anglais) (“Indonésie”)

Solheim, W.C. 1972, The "New look" of Southeast Asian prehistory. The Journal of the Siam Society, 60: 1-20. (en anglais) ("Le «nouveau regard» de la préhistoire de l'Asie du SudEst”)

Solheim, W.C., Bulbeck, D., \& Flavel, A. 2006, Archaeology and culture in Southeast Asia: Unravelling the Nusantao. The University of the Philippines Press, Diliman, Quezon City, 316 p. (en anglais) (“Archéologie et culture en Asie du Sud-Est : démêler le Nusantao") 
Sophady, H. 2014, The case of Phnom Teak Treang and Laang Spean cave, Cambodia: The potential for world heritage site nomination. Significance of the site for Huamn evolution in Asia, and the need for international cooperation. In: Human origin sites and the World Heritage Convention in Asia (Sanz, N., Ed.), World Heritage Papers Vol. 39, Heads Vol. 3, United Nations Educational, Scientific and Cultural Organization (UNESCO) Press, Paris: p. 164-178. (en anglais) ("Le cas de Phnom Teak Treang et de la grotte de Laang Spean, Cambodge : le potentiel pour la nomination du site au patrimoine mondial. L'importance du site pour l'évolution de Huamn en Asie, et le besoin de coopération internationale”)

URL: http://unesdoc.unesco.org/images/0022/002291/229174e.pdf

Sophady, H., Forestier, H., Zeitoun, V., Puaud, S., Frère, S., Celiberti, V., Westaway, K., Mourer, R., Mourer-Chauviré, C., Than, H., Billault, L., \& Tech, S. 2015, Laang Spean cave (Battambang province): A tale of occupation in Cambodia from the Late Upper Pleistocene to Holocene. Quaternary International, 1-15: 1-9. (en anglais) ("Grotte de Laang Spean (province de Battambang) : un récit d'occupation au Cambodge de la fin du Pléistocène supérieur à l'Holocène”) doi:10.1016/j.quaint.2015.07.049

Sørensen, P. 1976, Preliminary note on the absolute and relative chronology of two early palaeolithic site from Northern Thailand. In: Le Paléolithique inférieur et moyen en Inde, en Asie Centrale, en Chine et dans le Sud-Est asiatique, Proceedings of the IX $X^{\text {th }}$ UISPP Congress, Nice, Symposium VII (Ghosh, A.K., Ed.), Centre National de la Recherche Scientifique, Nice: p. 237-251. (en anglais) ("Note préliminaire sur la chronologie absolue et relative de deux sites paléolithiques du nord de la Thaïlande”)

Sharma, S. 2010, Southeast Asian elements in the archeological evidence of Northeast India. SPAFA Journal, 20: 19-30. (en anglais) ("Eléments de l'Asie du Sud-Est dans les données archéologiques de l'Inde du Nord-Est”)

Spennemann, D.H.R. 1991, Don't forget the bamboo. On recognising and interpretating butchery marks in tropical faunal assemblages. Some comments asking for caution. Tempus, 2: 108-134. (en anglais) ("N'oublie pas le bambou. Sur la reconnaissance et l'interprétation des marques de boucherie dans les assemblages fauniques tropicaux. Quelques commentaires demandant la prudence”)

Steenberg, A. 1980, New Guinea Gardens. A Study of Husbandry with Parallels in Prehistoric Europe. Academic Press, London, 222 p. (en anglais) (“Jardins de la Nouvelle-Guinée. Une étude sur l'élevage avec des parallèles dans l'Europe préhistorique”)

Tavoso, A. 1978, Le Paléolithique inférieur et moyen du Haut-Languedoc : gisement des terrasses alluviales du Tarn, du Dedou, de l'Agout, du Sor et du Fresquel. Etudes Quaternaires Vol. 5, Université de Provence, Marseille, 404 p. (in French) ("Lower and Middle Palaeolithic Upper Languedoc: Deposit of alluvial terraces from the Tarn, Dédou, Agout, Sor and Fresquel ")

Testart, A. 1977, Ethnologie de l'Australie et préhistoire de l'Asie du Sud-Est : évolution technique et milieu naturel. Journal de la Société des Océanistes, 54-55(33): 78-85. (in French) ("Australia's Ethnology and Prehistory of Southeast Asia: Technical Evolution and the Natural Environment")

U Aung Thaw 1971, The "Neolithic” culture of the Padah-lin caves. Asian Perspectives, 14: 123-33. (en anglais) (“La culture« néolithique » des grottes de Padah-lin”) 
Van Heekeren, H. 1972, The stone age of Indonesia $\left(2^{\text {nd }}\right.$ ed.). 's-Gravenhage, Martinus Nijhoff, 141 p. (in French) ("The Stone Age of Indonesia”)

West, J.A. 1989, A scanning electron microscopy study of bamboo knife and stone tool cutmarks. American Journal of Physical Anthropology, 78: 322-323. (en anglais) ("Une étude en microscopie électronique à balayage des couteaux en bambou et des marques de découpe d'outils en pierre”)

West, J.A., \& Louys, L. 2007, Differentiating bamboo from stone tool cut marks in the zooarchaeological record, with a discussion on the use of bamboo knives. Journal of Archaeological Science, 34: 512-518. (en anglais) ("Différencier les marques de découpe de bambou et de l'outil de pierre dans le corpus zooarchéologique, avec une discussion sur l'utilisation des couteaux en bambou”) doi:10.1016/j.jas.2006.06.007

White, J.C., \& Gorman, C. 2004, Patterns in “amorphous” industries: The Hoabinhian viewed through a lithic reduction sequence. In: Southeast Asian archaeology: Wilhelm G. solheim II Festschrift (Paz, V., Ed.), University of the Phillipines Press, Quezon City, p. 411-441. (en anglais) ("Les modèles dans les industries «amorphes» : le Hoabinhien vu à travers une séquence de réduction lithique”)

White, J.C., Lewis, H., Bouasisengpaseuth, B., Marwick, B., \& Arrell, K. 2009, Archaeological investigations in northern Laos: New contributions to Southeast Asian prehistory. Antiquity, Project Gallery, 83(319): online. (en anglais) ("Recherches archéologiques dans le nord du Laos : de nouvelles contributions à la préhistoire de l'Asie du Sud-Est”) URL: http://www.antiquity.ac.uk/projgall/white319/

White, J.C. 2011, Emergence of cultural diversity in mainland Southeast Asia: A review from Prehistory. In: Dynamics of human diversity: The case of Mainland Southeast Asia (Enfield, N.J., Ed.), Pacific Linguistics, Canberra: p. 9-46. (en anglais) ("L'émergence de la diversité culturelle en Asie du Sud-Est continentale : une revue de la Préhistoire”)

Wu, Y., Jiang, L. ;, Zheng, Y., Wang, C., \& Zhao, Z. 2014, Morphological trend analysis of rice phytolith during the early Neolithic in the Lower Yangtze. Journal of Archaeological Science, 49, 326-331. (en anglais) ("L'analyse des tendances morphologiques de phytolithes de riz au début du Néolithique dans le Bas Yangtze”) doi:10.1038/srep28136

Xhauflair, H. 2014, Plant use in the subsistence strategies of prehistoric hunther-gatherers in Palawan Island assessed from the lithic industry. Building up a reference collection. Thèse de doctorat du Muséum national d'Histoire Naturelle (MNHN), Paris, 626 p. (in French) ("L'utilisation des plantes dans les stratégies de subsistance des chasseurscueilleurs préhistoriques de l'île de Palawan évaluée par l'industrie lithique. Construire une collection de référence”)

Xhauflair, H., Pawlik, A., Gaillard, C., Forestier, H., Vitales, T.J., Callado, J.R., Tandang, D., Amano, N., Manipon, D., \& Dizon, E. 2016, Characterisation of the use-wear resulting from bamboo working and its importance to address the hypothesis of the existence of a bamboo industry in prehistoric Southeast Asia. Quaternary International, 416: 95-125. (en anglais) ("Caractérisation de l'usure résultant du travail du bambou et son importance pour répondre à l'hypothèse de l'existence d'une industrie du bambou dans l'Asie du Sud-Est préhistorique”) doi:10.1016/j.quaint.2015.11.007 
Xueping, J., Kuman, K., Clarke, R.J., Forestier, H., Li, Y., Ma, J., Qiu, K., Li, H., Wu, Y., Xie, H., \& Dequan, H. 2016, The oldest Hoabinhian technocomplex in Asia (>43.5ka) at Xiaodong rockshelter, Yunnan Province, Southwest China. Quaternary International, 400: 166-174. (en anglais) ("Le plus ancien technocomplex Hoabinhien en Asie (> 43,5 ka) dans l'abri sous roche de Xiaodong, province du Yunnan, Chine du Sud-Ouest”) doi:10.1016/j.quaint.2015.09.080

Yi, S., Lee, J.J., Kim, S., Yoo, Y., \& Kim, D. 2008, New data on the Hoabinhian: Investigations at Hang Cho cave, Northern Vietnam. Bulletin of Indo-Pacific Prehistory Association, 28: 73-79. (en anglais) ("Nouvelles données sur le Hoabinhien : Recherches à la grotte de Hang Cho, Nord du Vietnam")

Zeitoun, V. 2004, Réconciliation des modèles « évolution régionale et sortie d'Afrique ». In: Paléoécologie Paleoecology: General sessions and posters (Le Secrétariat du Congrès, Eds.), BAR Vol. S1271, Hadrian Books, Oxford: p. 49-58. (in French) ("Reconciliation of "regional evolution and exit from Africa" models")

Zeitoun, V. 2009, The Human canopy. Homo erectus, Homo soloensis, Homo pekinensis and Homo floresiensis. B.A.R. Vol. S1937, Hadrian Books, Oxford, 177 p. (en anglais) ("La canopée humaine. Homo erectus, Homo soloensis, Homo pekinensis et Homo floresiensis")

Zeitoun, V., Détroit, F., Grimaud-Hervé, D., \& Widianto, H. 2010, Solo man in question: Convergent views to split Indonesian Homo erectus in two categories. Quaternary International, 223-224: 281-292. (en anglais) (“L’Homme en question : des vues convergentes pour diviser l'Homo erectus indonésien en deux catégories”) doi:10.1016/j.quaint.2010.01.018

Zeitoun, V., Forestier, H., Pierret, A., Chiemsiouraj, C., \& Lorvankham, M. 2012a, Multimillenary occupation in Northwestern Laos: Preliminary results of excavations at the Ngeubhinh Mouxeu rock-shelter. Comptes Rendus Palevol, 11: 305-313. (en anglais) (“Occupation multimillénaire dans le nord-ouest du Laos : résultats préliminaires des fouilles de l'abri de Ngeubhinh Mouxeu”) doi:10.1016/j.crpv.2011.11.001

Zeitoun, V., Forestier, H., Auetrakulvit, P., Rasse, M., Khaokhiew, C., Rasse, M., Davtian, G., Winayalai, \& Tiamtinkrit, C. 2012b, Discovery of the prehistoric site at Sao Din (Nanoi, Nan Province, Northern Thailand): Stone tools and new geological insights. Comptes Rendus Palevol, 11: 575-580. (en anglais) ("Découverte du site préhistorique à Sao Din (Nanoi, province de Nan, nord de la Thaïlande) : outils en pierre et nouvelles connaissances géologiques”) doi:10.1016/j.crpv.2012.06.007

Zeitoun, V., Forestier, H., \& Nakbunlung, S. 2008, Préhistoires au sud du Triangle d'Or. Éditions IRD, Paris, 252 p. (in French) ("Prehistories south of the Golden Triangle") 


\title{
The Hoabinhian techno-complex in Mainland Southeast Asia: The history of a pebble which hides the forest
}

\author{
Hubert Forestier ${ }^{1}$, Heng Sophady ${ }^{2}$, Vincenzo Celiberti ${ }^{3}$ \\ 1. Muséum national d’Histoire naturelle, UMR 7194 CNRS-MNHN-UPVD, Institut de Paléontologie Humaine, \\ 1, rue René-Panhard, 75013 Paris, France. Email: hubforestier@gmail.com \\ 2. Ministry of Culture and Fine Arts, 227 Kbal Thnal, Preah Norodom Boulevard, Sangkat Tonle Bassac, Khan \\ Chamkar Mon Phnom Penh 12305, Cambodia. Email: hsophady@yahoo.com \\ 3. Université de Perpignan Via Domitia, UMR 7194 CNRS-MNHN-UPVD, Centre Européen de Recherches \\ Préhistoriques, Avenue Léon-Jean Grégory, 66720 Tautavel, France. \\ Email: vincenzo.celiberti@cerptautavel.com
}

\begin{abstract}
:
The prehistory of Southeast Asia is characterized by some chaînes opératoires which are still not very well known or poorly described. This lack of knowledge comes on the geographical remoteness of these tropical regions with respect to Western prehistoric problems developed during the past two centuries. The prehistory of the Far East is complex, original, surprising because on the sidelines of major technical lineages elsewhere known to the relevant period, the one of anatomically modern human advent. This regional prehistory of about 2 million $\mathrm{km}^{2}$, which is called Peninsular or Continental Southeast Asia, refers to the development of a science in motion whose construction is still relevant. In colliding with the classic thesis of evolution and technical progress, the Hoabinhian shakes the rules and landmarks in prehistory. It is located at the antipodes of the classical model (Eurasian, African) of development of the stone tools according to the improvement and gradual lightening of the tool-kit, from the pebble culture until the Neolithic. Our reflection is precisely on this singularity, about a unique technical phenomenon that remains difficult to place on the evolutionary axis of industries as it exists elsewhere in the world or in East Asia (China, Korea, Japan). The regularity and homogeneity of cobble-based tool shapes in a vast area and for a record length of nearly 30,000 years are the main features of this unorthodox technocomplex that questions the cognitive capacity of Homo sapiens in a wet tropical ecosystem. But we also question the nature of the existing links between prehistoric men and their lithic productions, and the role played by knapped stone techniques during the human development in this region away from Europe. To this monotonous longevity of pebble-based tools is added the absence of pointed lithic tools (tips, apical ends, tool with converging salient edges, etc.) as they are found everywhere else in hunter-gatherer groups, whether from the Upper Palaeolithic or historical times.

This is why the stability of these pebble-based tools would hide a whole range of complexity unknown to the technical field such as the elusive activities relating to the transformation of hard animal materials but also of vegetable materials not preserved in archaeological context. The thought process from the mineral towards the question of the vegetal sends back the need to complete the "toolbox" of Hoabinhian prehistoric artisans with sharp objects. Known to date only through the ethnographic data, the "vegetal civilization" leads naturally to reflect on the importance of this perishable material in the tool-kit of the last hunter-gatherers from the Upper Pleistocene rain forest in continental Southeast Asia. In other words, the possibility of another technical existence in equilibrium with the external environment.

After having highlighted the originality of Hoabinhian cultural phenomenon compared with impact of research in palaeoanthropology and prehistory in the Southeast Asia regions, this paper will
\end{abstract}


present from a strictly qualitative point of view the main chaînes opératoires that are present within the Hoabinhian techno-complex, a regional variant that characterizes the main culture of Southeast Asia Final Palaeolithic hunters-gatherers between about 30000 and 5000 years BP. More generally, details will be provided on the informative incompleteness of the lithic phenomenon as archaeological data and, also, on its overcoming as a phenomenon. It will therefore be a question of rethinking the reverse of the lithic-lignic dialectic, that is to say the vegetable objects forever extinguished, in the light of stone tools, the only preserved markers of time, technique, space and absence.

Keywords: Southeast Asia; Late Upper Palaeolithic; Hoabinhian; chaîne opératoire; pebble-tool; hunter-gatherer; anatomically modern humans 